\title{
Article \\ Genome-Wide In Silico Identification and Comparative Analysis of Dof Gene Family in Brassica napus
}

\author{
Neeta Lohani (D), Saeid Babaei, Mohan B. Singh and Prem L. Bhalla * (D) \\ Plant Molecular Biology and Biotechnology Laboratory, Faculty of Veterinary and Agricultural Sciences, \\ The University of Melbourne, Parkville, Melbourne, VIC 3010, Australia; nlohani@student.unimelb.edu.au (N.L.); \\ sbabaeighara@student.unimelb.edu.au (S.B.); mohan@unimelb.edu.au (M.B.S.) \\ * Correspondence: premlb@unimelb.edu.au; Tel.: +61-3-8344-9651
}

check for

updates

Citation: Lohani, N.; Babaei, S.;

Singh, M.B.; Bhalla, P.L.

Genome-Wide In Silico Identification

and Comparative Analysis of Dof

Gene Family in Brassica napus. Plants

2021, 10, 709. https://doi.org/

10.3390/plants10040709

Received: 28 February 2021

Accepted: 6 April 2021

Published: 7 April 2021

Publisher's Note: MDPI stays neutral with regard to jurisdictional claims in published maps and institutional affiliations.

Copyright: (C) 2021 by the authors. Licensee MDPI, Basel, Switzerland. This article is an open access article distributed under the terms and conditions of the Creative Commons Attribution (CC BY) license (https:/ / creativecommons.org/licenses/by/ $4.0 /)$.

\begin{abstract}
DNA binding with one finger (DOF) proteins are plant-specific transcription factors that play roles in diverse plant functions. However, little is known about the DOF protein repertoire of the allopolyploid crop, Brassica napus. This in silico study identified 117 Brassica napus Dof genes (BnaDofs) and classified them into nine groups (A, B1, B2, C1, C2.1, C2.2, C3, D1, and D2), based on phylogenetic analysis. Most members belonging to a particular group displayed conserved gene structural organisation and protein motif distribution. Evolutionary analysis exemplified that the divergence of the Brassica genus from Arabidopsis, the whole-genome triplication event, and the hybridisation of Brassica oleracea and Brassica rapa to form B. napus, followed by gene loss and rearrangements, led to the expansion and divergence of the Dof transcription factor (TF) gene family in B. napus. So far, this is the largest number of Dof genes reported in a single eudicot species. Functional annotation of BnaDof proteins, cis-element analysis of their promoters, and transcriptomic analysis suggested potential roles in organ development, the transition from the vegetative to the reproductive stage, light responsiveness, phytohormone responsiveness, as well as potential regulatory roles in abiotic stress. Overall, our results provide a comprehensive understanding of the molecular structure, evolution, and possible functional roles of Dof genes in plant development and abiotic stress response.
\end{abstract}

Keywords: Dof; Brassica napus; canola; transcription factor; polyploidy; abiotic stress

\section{Introduction}

Brassica napus, the second-largest economically important oilseed crop, is used as edible oil and livestock forage, and in the pharmaceuticals, cosmetics, and biofuel industries [1]. The yield of B. napus is constrained by harsh environmental conditions such as drought, extreme temperature, and salinity [2]. Dissecting the evolution and function of diverged plant-specific transcription factor (TF) families such as DNA binding with one finger (DOF) is required for gaining fundamental knowledge about the mechanisms underlying stress responses in B. napus and for developing stress-tolerant varieties for climate-smart agriculture.

The DOF TFs are plant-specific transcription factors, first identified in maize in 1995, and were shown to play an essential role in regulating carbon metabolism-related and lightregulated genes [3-5]. Subsequently, a diverse number of DOF TFs have been identified in several plants, including 36, 30, and 96 in Arabidopsis, rice, and wheat, respectively [6-8]. In Arabidopsis, the first protein-protein interaction of DOF domain protein with bZIP protein associated with the stress response was reported, indicating the potential role of DOF TFs in complex regulatory networks [9].

DOF TFs are typically composed of 200-400 amino acid residues with a variable C-terminal region and are mainly characterised by a highly conserved DNA-binding domain, i.e., the DOF domain, located towards the N-terminal of the protein $[10,11]$. The DOF domain consists of only one Cys2/Cys2 zinc finger structure, which includes about 
52 amino acid residues, that specifically recognises and binds a cis-regulatory element $\left(5^{\prime}-\mathrm{T} / \mathrm{AAAAG}-3^{\prime}\right)$ in the gene promoters [12]. Another specific feature of DOF TFs is a bipartite nuclear localisation signal (NLS) comprised of two short basic flanking regions (B1 and B2) with a 17 amino acid spacer. The bipartite NLS is highly conserved and plays a vital role in directing DOF TFs to the cell nucleus [13].

Phylogenetic studies suggest a common ancestor (conserved as a single copy in Chlamydomonas) of Dof genes, which through numerous rounds of gene duplication drove the structural and functional diversification of the Dof gene family [12]. This evolutionary diversification may be related to acquiring new specialised functions needed to adjust and adapt to diverse and complex plant growth conditions. Brassica napus, an amphidiploid (AACC, $2 \mathrm{n}=4 \mathrm{x}=38$ ), originated from a natural crossing between two ancestral diploid parents, Brassica oleracea (CC, $2 \mathrm{n}=2 \mathrm{x}=18$ ) and Brassica rapa (AA, $2 \mathrm{n}=2 \mathrm{x}=20$ ), approximately 7500 years ago [14]. Brassica species belonging to the Brassica genus and Brassicaceae family offer a valuable model for studying polyploid genome evolution, mechanisms associated with gene duplications, loss of duplicated genes and gene neo- and sub- functionalisation $[15,16]$. The availability of $B$. napus, B. rapa, and B. oleracea genome sequences has provided an exceptional opportunity to identify and characterise key genes from a genome-wide perspective [14,16,17].

This study aims to provide a complete description of the Dof gene family in B. napus by performing a genome-wide in silico identification, characterisation, and evolutionary and functional analysis of the Dof gene family. We report identifying one hundred seventeen genes as members of the Dof transcription factor family from B. napus, belonging to nine groups. We also performed a detailed analysis of the Dof genes in terms of physical properties of proteins, chromosomal location, gene structure, motif analysis and phylogenetic relationships, and gene duplication. Orthology and synteny analysis was also carried out to explore the evolutionary history and divergence of the Dof TFs family in B. napus, B. rapa, B. oleracea, and Arabidopsis. Furthermore, functional annotation and cis-acting regulatory element analysis of BnaDof gene promoters and their expression profiles highlighted their potential roles in regulating distinct and diverse developmental processes and stress responses.

\section{Results}

\subsection{Identification and Characterisation of BRASSICA Napus Dof Gene Family}

To identify the Dof TF family genes in B. napus, we carried out BLASTP searches using DOF-domain search model accession (Pfam: PF02701), searched the PlantTFDB4.0, and finally retrieved and analysed 156 amino acid sequences using the SMART8.0 database [18-20]. We identified 117 full-length $B$. napus genes as putative members of the Dof gene family from the Brassica napus reference genome (Brassica_napus. annotation_v5). We assigned new identifiers to the 117 B. napus Dof genes by using the prefix "Bna" for B. napus, followed by "Dof" and a number based on their chromosomal locations (Figure 1). Thus, the identified B. napus Dof family members were named BnaDof01 to BnaDof117 (Table S1). The BnaDofs were distributed on all 19 chromosomes, with 60 BnaDofs located on A genome (48 on chromosomes A01-A10), 56 situated on C genome (40 on chromosomes C01-C09), and one gene placed on an unknown chromosome (BnaDof117; BnaUnng03510D). Chromosome C03, being the largest, included the most BnaDofs (11 BnaDofs), followed by A09 with 10 BnaDofs. Out of 117 BnaDof genes, the exact chromosomal location was unknown for the 58 genes located on A03_random, A04_random, A05_random, A07_random, A09_random, A10_random, Ann_random, C01_random, C03_random, C05_random, C06_random, Cnn_random, and Unn_random chromosomes. 

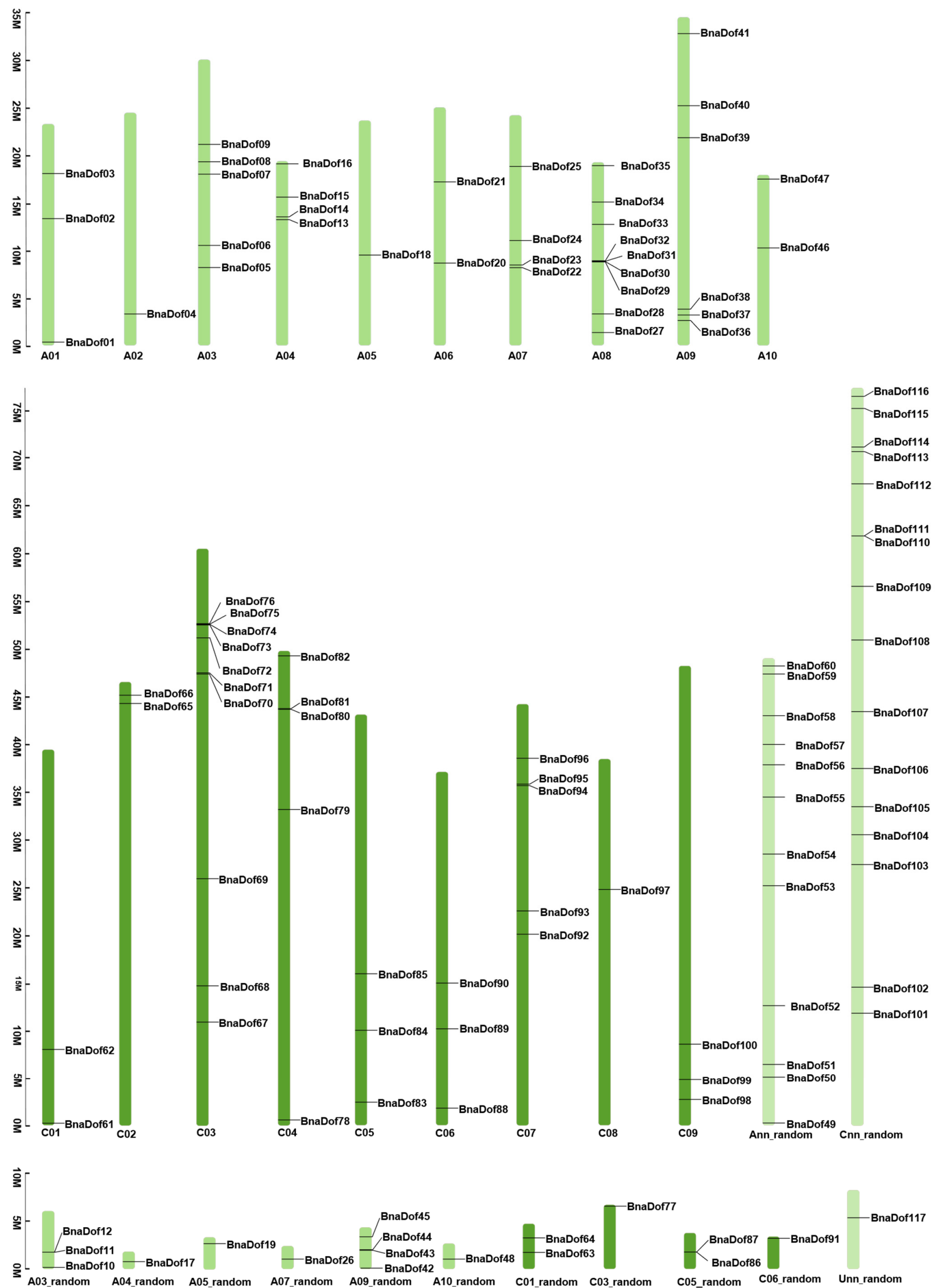

Figure 1. Chromosomal mapping and frequency distribution of Brassica napus DNA binding with one finger (DOF) transcription factors (BnaDofs01-117). 
The physical and chemical properties of BnaDof proteins are outlined in Table S1. The size of the BnaDof protein sequences ranged from 77 (BnaDof30, BnaDof31, BnaDof75, and BnaDof76) to 453 (BnaDof33) amino acids. All BnaDofs had a marginally higher percentage of aliphatic amino acids than aromatic amino acids. The predicted isoelectric point (pI) ranged from 4.78 (BnaDof20) to 10.14 (BnaDof30, BnaDof31, BnaDof75, and BnaDof76), and the molecular weight (MW) ranged from $8.76 \mathrm{kD}$ (BnaDof30, BnaDof31, BnaDof75, and BnaDof76) to $50.33 \mathrm{kD}$ (BnaDof33). The Grand Average of Hydropathy (GRAVY) values of the BnaDofs reflected the hydrophilic nature of these proteins.

We also identified $62 \mathrm{~B}$. oleracea genes as members of the Dof TF family (Table S2). In B. rapa, Ma et al. [21] reported 76 BnaDof genes. However, among B. rapa Dof genes, Bra007632 contained a B3 DNA binding domain, auxin response factor domain, and Aux/IAA domain in addition to the DOF domain. Bra007632 was orthologous to AUXIN RESPONSE FACTOR 18 (ARF18, At3g61830), and hence, we excluded it from our analysis. It is worth noting that in addition to the 117 BnaDof genes, one other gene (BnaA07g02590D) showed the presence of the DOF domain along with a Syntaxin domain. Further investigation revealed that this gene was homologous to Bra002057, and reported as a B. rapa Dof gene family member [21]. However, this gene is orthologous to Arabidopsis SYNTAXIN OF PLANTS 21 (SYP21, At5g16830). We decided to exclude BnaA07g02590D and Bra002057 from our analysis as these genes can be classified as members of the SNAP Receptor (SNARE) protein family [22]. Thus, finally, we considered 74 Dof genes from B. rapa, 62 Dof genes from B. oleracea, and 117 Dof genes from B. napus.

\subsection{Phylogenetic Relationships of the Dof Gene Family in B. napus}

We explored the phylogenetic relationships between the Dof families in B. napus and Arabidopsis by first performing alignment of the 117 BnaDofs and 36 Arabidopsis Dofs using CLUSTALW [23]. The multiple sequence alignment was then used to construct the unrooted phylogenetic tree in MEGA7.0 [24] using the maximum likelihood method with 500 bootstrap values (Figure 2). We then classified BnaDofs into four major groups: A, B, $\mathrm{C}$, and D and the following nine subgroups: A, B1, B2, C1, C2.1, C2.2, C3, D1, and D2, based on the phylogenetic tree. The largest group was C, with $35 \%$ of BnaDofs, followed by group D with $28 \%$ BnaDofs. Among the subgroups, D2 was the largest, comprising 18\% BnaDofs. The least number of BnaDofs belonged to subgroup C1.

We further explored the phylogenetic relationship between the Dof gene family in B. napus, B. oleracea, B. rapa, and Arabidopsis. As mentioned earlier, there were 76 (74 included in our analysis) B. rapa Dofs and 36 Arabidopsis Dofs, and we identified 117 and 62 Dof genes in B. napus and B. oleracea, respectively [6,21]. Subsequently, a total of 289 DOF protein sequences were utilised to construct the phylogenetic tree. Based on the resulting phylogenetic tree, the Dof gene family can be classified into four major groups and nine subgroups, as described earlier (Figure S1). The tree illustrates the expansion and divergence of the Dof gene family from Arabidopsis to B. napus. A few genes from the two diploid progenitors were lost in B. napus during hybridisation, such as Bra010136 and Bra013490 from B. rapa, and Bo3g106920 Bo01143s010 from B. oleracea, with no orthologs in Brassica napus. A few $B$. rapa genes underwent further duplications after hybridisation; for example, Bra020880 had four orthologs in B. napus (BnaDof30, BnaDof31, BnaDof75, and BnaDof 76), and Bra023888 had two orthologs in B. napus (BnaDof03 and BnaDof64). However, the majority of BnaDofs were consistently inherited from their progenitors (Table S3). Based on the known chromosomal locations, we confirmed a total of 42 and 33 gene pairs which maintained their relative positions between the $B$. rapa genome and $A_{n}$ sub-genome in the $B$. napus and B. oleracea genomes and $C_{n}$ sub-genome in B. napus, respectively. 


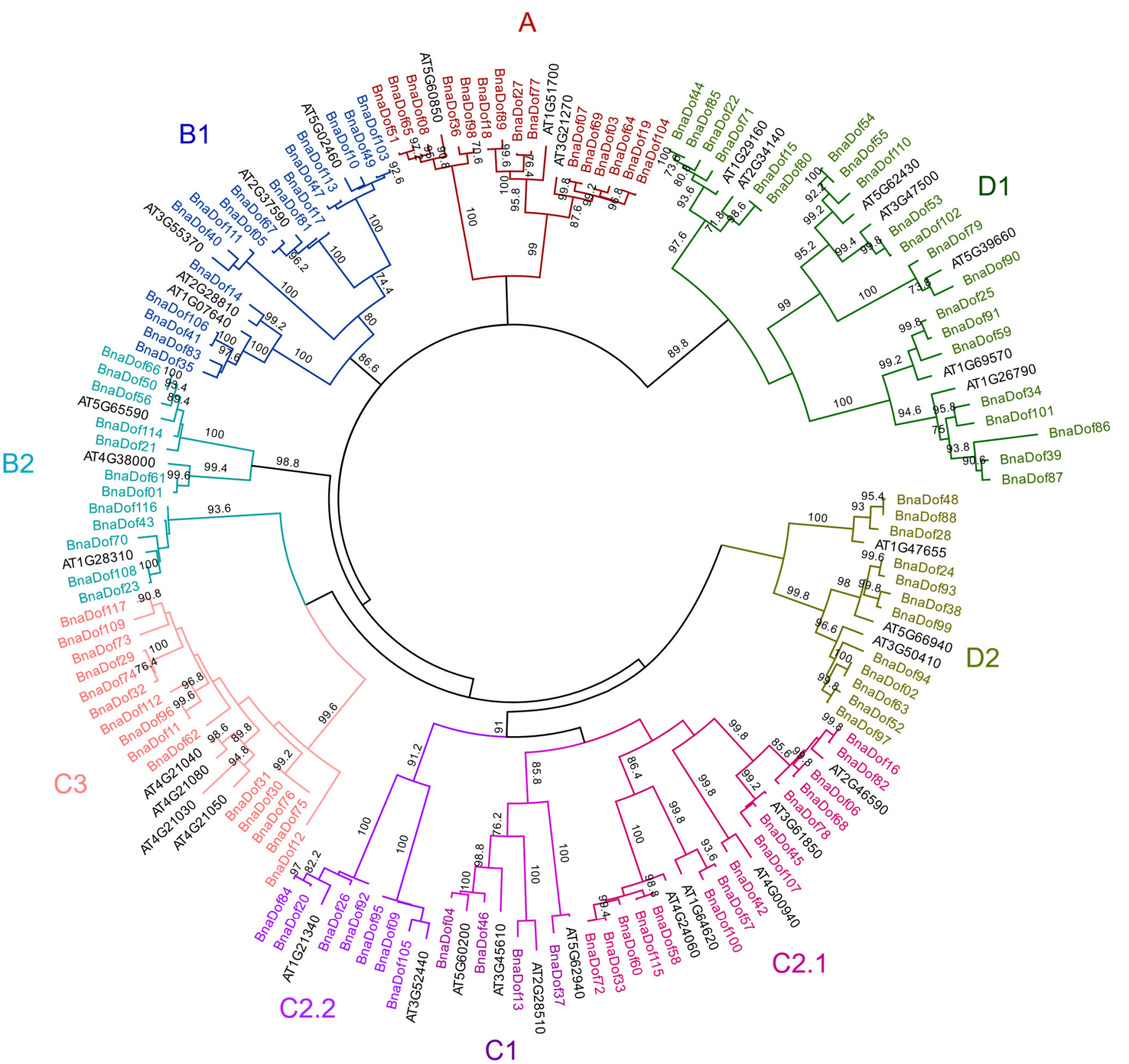

0.3

Figure 2. Phylogenetic tree of B. napus and Arabidopsis DOF proteins. The evolutionary history was inferred by using the maximum likelihood method based on the JTT (Jones-Taylor-Thornton) matrix-based method. Maximum-likelihood bootstrap values (500 replicates) above $70 \%$ are shown. The analysis involved 153 amino acid sequences. Phylogenetic topology was generated via MEGA7.

\subsection{Gene Structure and Conserved Motifs of BnaDofs}

To gain further understanding of the structural diversity of BnaDofs, we studied exon-intron organisation and identified the presence of conserved protein motifs (Figure 3). The analysis revealed 61 BnaDofs with no introns, whereas 46, 8, and 2 BnaDofs had one, two, and three introns, respectively. The majority of the genes within a given subgroup showed a similar exon-intron organisation. For example, all the $\mathrm{C} 1$ subgroup BnaDofs had one intron, and most members of subgroups D2, A, B2, C3, and C2.2 had no introns. Similarly, BnaDofs belonging to subgroup B1 had at least one intron. The most diverse 
gene structure organisation was observed in the members of subgroup D1, ranging from zero to three introns.

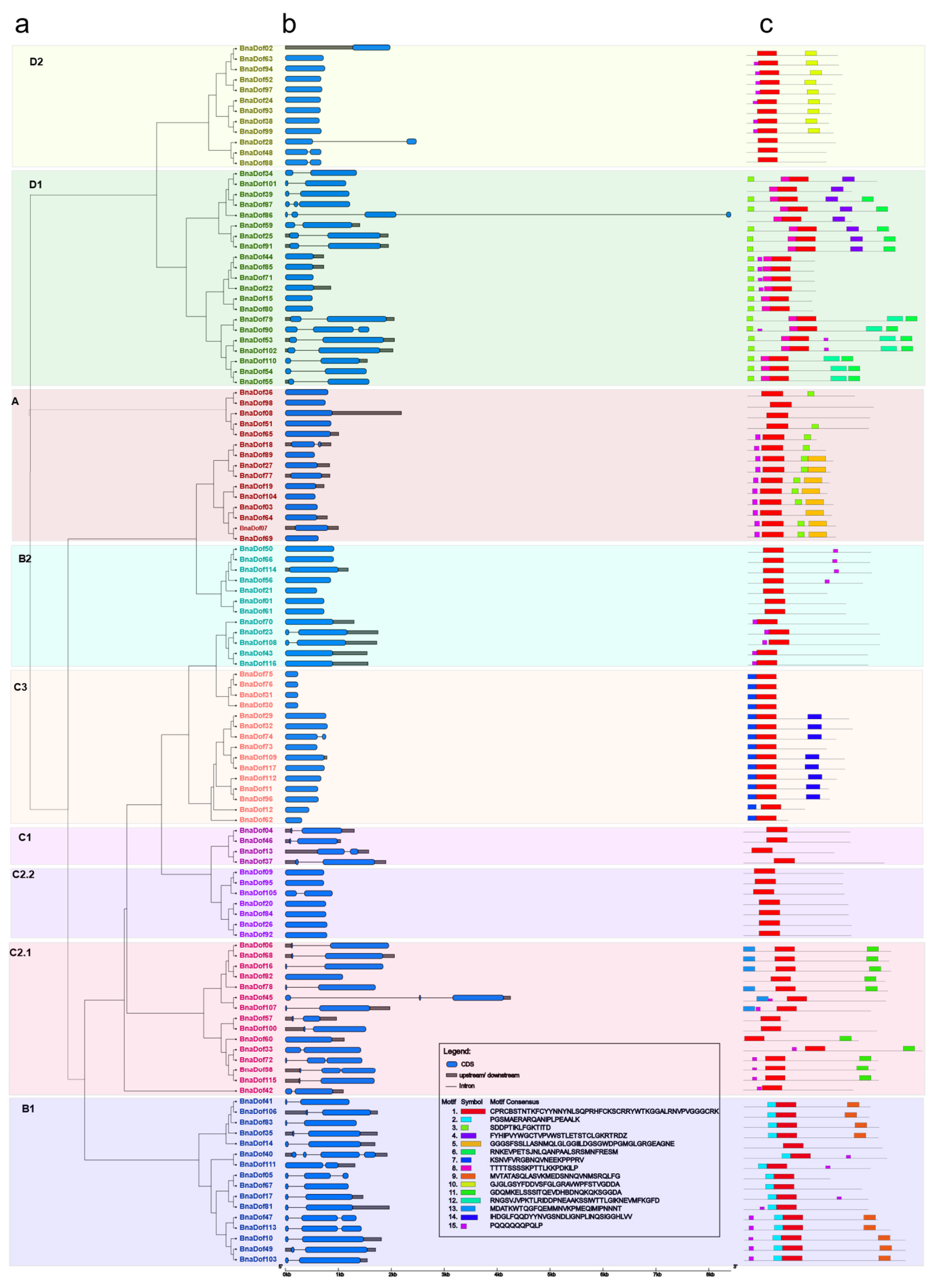

Figure 3. Phylogenetic relationships, gene structure, and conserved protein motifs in the Dof gene family members in B. napus. (a) Phylogenetic relationships of BnaDofs. The phylogenetic tree was constructed with MEGA 7.0 using the neighbour-joining (NJ) method with 1000 bootstrap replicates and the Poisson correction method. The nine Dof groups are displayed in different text colours and enclosed in the respective colour boxes. (b) Gene structure of BnaDof genes. Grey boxes indicate untranslated 5' - and 3'-regions; blue boxes indicate exons; and black lines indicate introns. Scale bar represents gene length. (c) Distribution of conserved motifs in BnaDof proteins. The sequence of each motif (1-15) displayed in different coloured boxes is provided in the legend. 
Motif analysis performed using MEME showed a highly conserved motif, motif 1 , representing the DOF-type domain, across all the 117 BnaDof amino acid sequences [25]. The conserved nature of motif distribution within the BnaDof protein subgroup also highlighted their phylogenetic relationships. It is worth mentioning that conserved motif distribution also existed among members of different clades within a subgroup. For example, BnaDof28, BnaDof48, and BnaDof88, which belonged to one clade in subgroup D2, showed only one out of three conserved motifs found in the other D2 subgroup members. Thus, the results suggest that gene and protein structural divergence across subgroups probably governs the functional diversity in the Dof subgroups.

\subsection{Orthologous Gene Clustering of Dof Gene Family in B. napus, B. oleracea, B. rapa, and Arabidopsis}

To understand the evolutionary relationships of the Dof gene family among the four important members of the Brassicaceae family-B. napus, B. oleracea, B. rapa, and Arabidopsis, we carried out an orthology analysis in OrthoVenn2 web platform [26]. The identified orthologous clusters in the four species are illustrated in Figure 4. 134 DOF proteins from all four species were clustered in 29 orthologous groups. We also identified 25 Brassica-specific clusters with 88 DOF proteins from the three Brassica species. One Arabidopsis DOF protein (AT3G45610), 17 BnaDofs, and 6 B. rapa DOFs did not cluster in any orthologous group and were identified as singletons. No singletons were identified in B. oleracea. Furthermore, there were seven clusters (17 DOFs) between B. rapa and B. napus, six clusters (12 DOFs) between B. oleracea and B. napus and two clusters between $B$. rapa and $B$. oleracea. The absence of $B$. napus genes in $B$. rapa-B. oleracea-specific clusters suggests that genes belonging to these clusters might have been lost during the hybridisation event. A detailed list of orthologous gene clusters and singletons is provided in Table S4a,b.

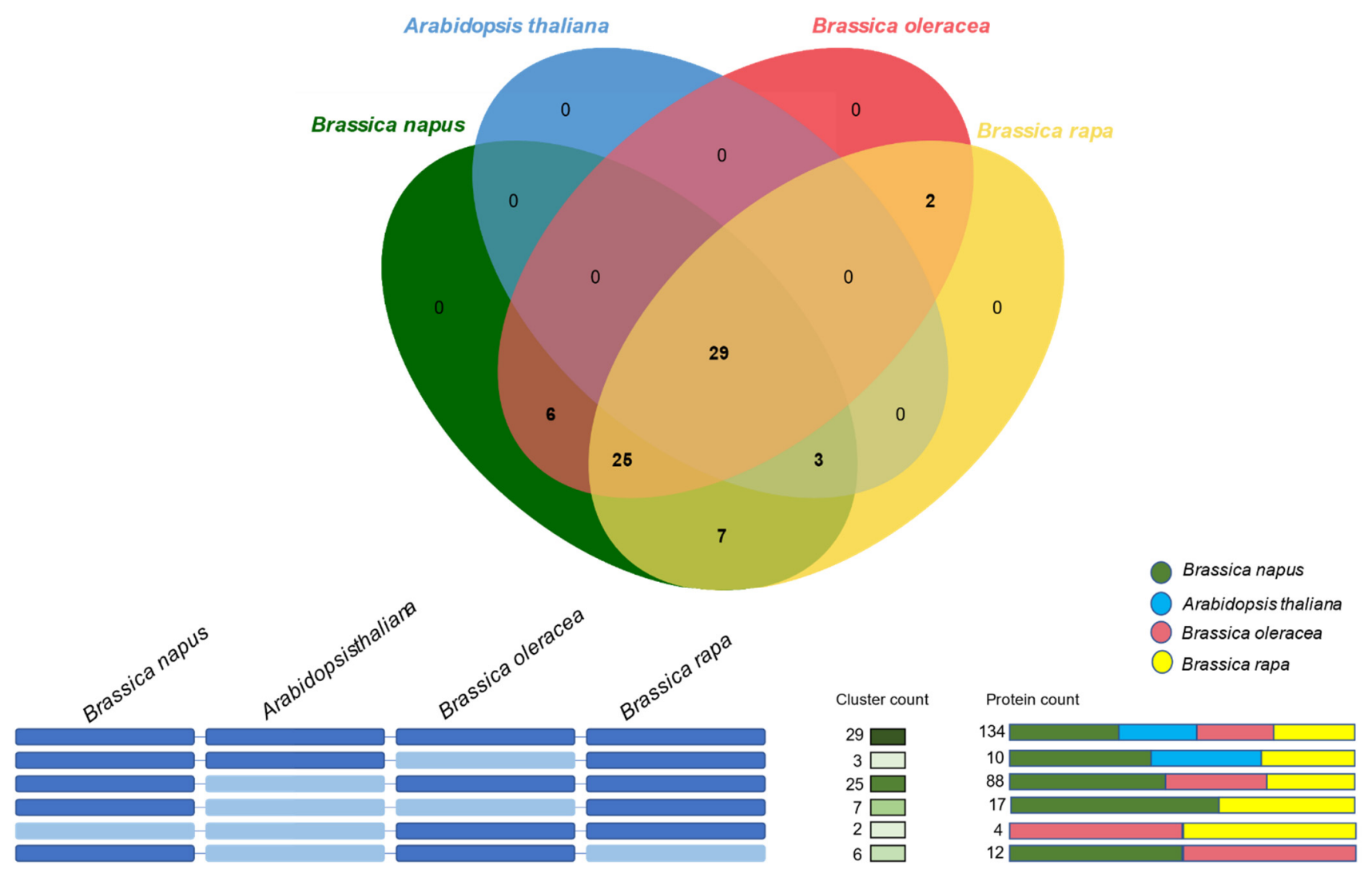

Figure 4. Orthologous gene clustering analysis. The orthologous gene clusters between the Dof gene family in B. napus, B. oleracea, B. rapa, and Arabidopsis were identified and visualised using the OrthoVenn2 web platform. The e-value cut-off $1 \mathrm{e}-10$ was used for the analysis. 


\subsection{Evolution and Divergence of BnaDofs}

The expansion of a gene family occurs because of duplication events arising at a wholegenome or small scale. We first identified duplicated gene pairs among BnaDofs based on the sequence similarity (Table S5a). We found 128 gene pairs with $>80 \%$ sequence similarity. Gene pairs were identified as tandemly duplicated if the new gene/sequence was found adjacent (within a $100 \mathrm{~kb}$ window) to the duplicated genomic region. Based on these criteria, three BnaDof gene pairs were identified as tandemly duplicated (BnaDof30-31, BnaD of 75-76, and BnaD of 86-87). The rest of the BnaDof gene pairs underwent interspersed duplications. Furthermore, BLASTP- and MCScanX-based methods identified 85 segmental, 26 dispersed, 4 tandem, and 1 proximal duplication event (Table S5b) [20,27]. These results highlight that segmental duplication events played a critical role in shaping the Dof gene family in B. napus.

Nucleotide substitutions producing an amino acid change are termed non-synonymous, and those that do not are termed synonymous. The ratio of non-synonymous to synonymous substitutions $(\mathrm{Ka} / \mathrm{Ks})$ in a protein-coding gene reflects the magnitude and direction of selection pressure acting on a protein sequence [28]. A Ka/Ks value $<1$ indicates that a gene pair has experienced negative or purifying selection (acting against change), whereas $\mathrm{Ka} / \mathrm{Ks}>1$ indicates positive or adaptive selection (driving change), and $\mathrm{Ka} / \mathrm{Ks}=1$ indicates neutral selection [29]. Thus, we calculated the Ka/Ks ratio among the duplicated gene pairs (Table S5a). Among the 128 identified duplicated BnaDof gene pairs, six gene pairs (BnaDof30 and BnaDof31, BnaDof30 and BnaDof75, BnaDof13 and BnaDof75, BnaDof30 and BnaDof 76, BnaDof31 and BnaDof76, and BnaDof75 and BnaDof76) were 100\% identical, and their Ka $=\mathrm{Ks}=0$. Three gene pairs (BnaDof29 and BnaDof74, BnaDof32 and BnaDof74, and BnaDof72 and BnaDof33) underwent positive or adaptive selection $(\mathrm{Ka} / \mathrm{Ks}>1)$, and the remaining 119 gene pairs experienced negative or purifying selection $(\mathrm{Ka} / \mathrm{Ks}<1)$. Purifying selection plays a potential role in maintaining the conservation of the Dof genes structure during evolution. The gene pairs undergoing positive selection indicate the presence of mutations that might be advantageous for B. napus.

The syntenic relationships between chromosome segments of different species can provide valuable insights into the origin of the gene family members. For the synteny analysis, only the genes with known chromosomal locations were considered. We performed a syntenic analysis between Dof genes from B. napus and Arabidopsis (Figure 5a). Following our orthology analysis, we identified orthologs for 35 Arabidopsis genes in $B$. napus. In addition, we also constructed a synteny map between Dof genes from B. napus, B. oleracea, and B. rapa. 58 out of 117 genes in B. napus and two out of 62 genes in B. oleracea were discarded due to uncertain chromosomal locations (Figure $5 b$ ). Out of the remaining 59 BnaDofs, $98.3 \%$ of genes were placed in collinear blocks. In B. rapa (42/75 Dof genes) and in B. oleracea (47/60 Dof genes), 56 and $78.3 \%$ were placed in collinear blocks, respectively. Since the same gene in $B$. napus could be in a collinear block relative to $B$. rapa but not relative to $B$. oleracea, the numbers reported for $B$. rapa and B. oleracea indicated how many genes were collinear with the corresponding orthologs in B. napus. For B. napus, instead, this number reported the number of collinear genes in at least one of the other species. 
a

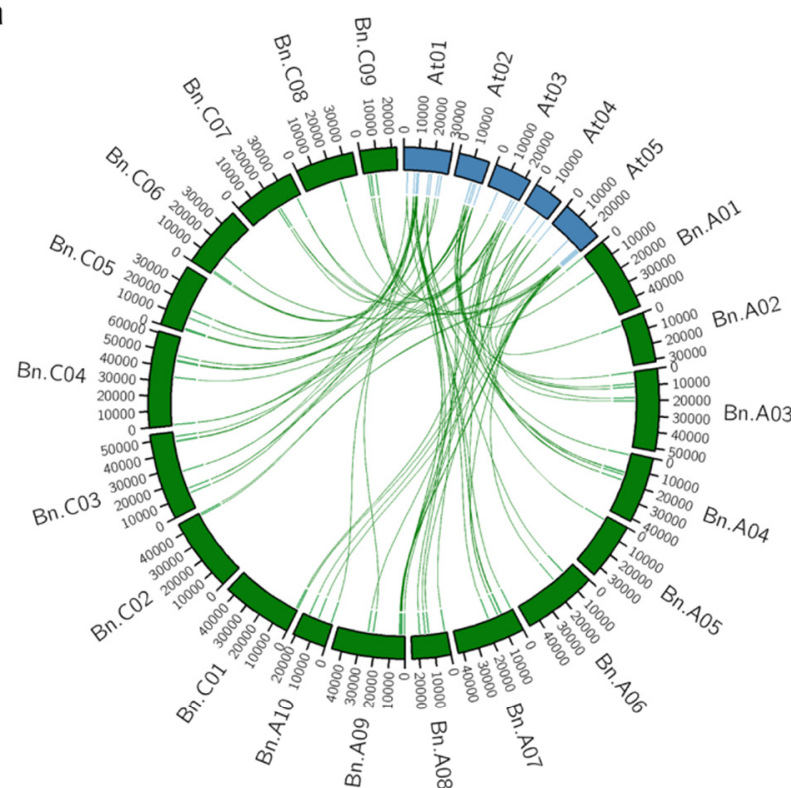

C

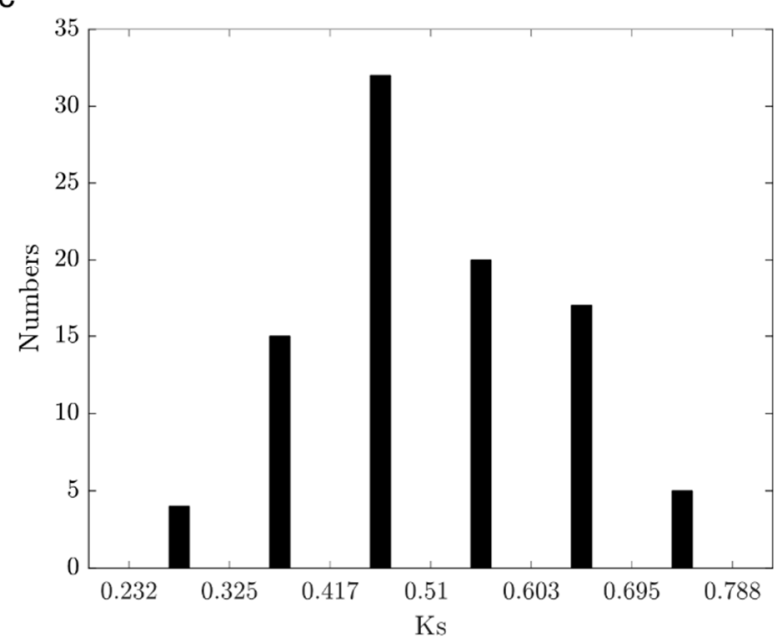

b

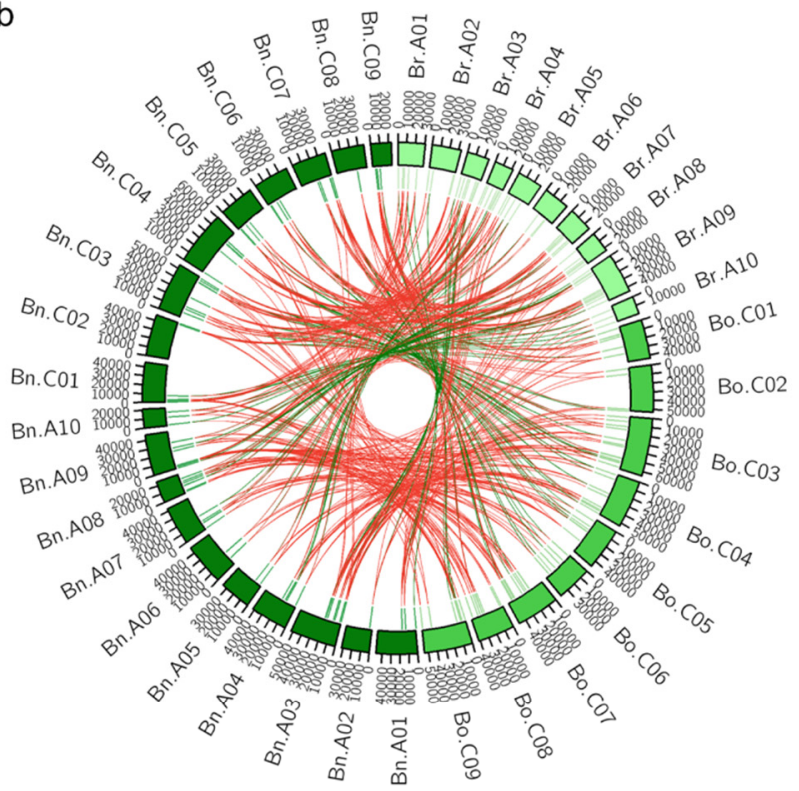

d

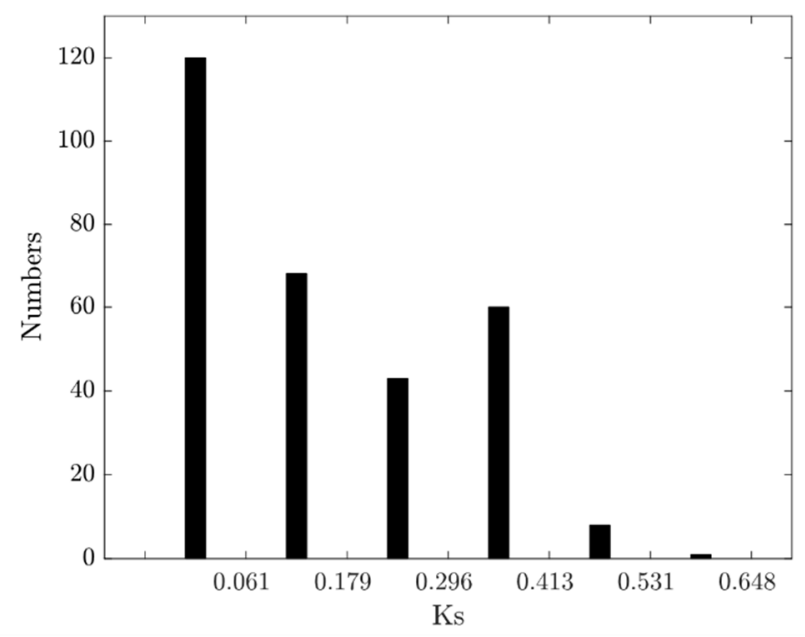

Figure 5. Synteny analysis (a) Synteny of Dof gene family between Arabidopsis thaliana and B. napus. Ideograms of chromosomes of $A$. thaliana (blue) and B. napus (dark green) are displayed in the outer circle, while in the inner circle indicates the positions of the genes in the corresponding chromosomes. Green links connect orthologous genes between the two species. The chromosome scale is in Kb. At: Arabidopsis thaliana; Bn: Brassica napus. (b) Synteny of DOF transcription factors within the Brassicaceae genus. Ideograms of chromosomes of B. rapa (pale green), B. oleracea (lime green), and B. napus (dark green) are displayed in the outer circle, while the inner circle indicates the positions of the genes in the corresponding chromosomes. Green links connect collinear orthologous genes, while red links connect orthologues genes that underwent chromosomal translocation events. The chromosome scale is in $\mathrm{Kb}$. Bn: Brassica napus; Bo: Brassica oleracea; Br: Brassica rapa. (c) Density of Ks values of Dof orthologous gene pairs between B. napus and Arabidopsis. Analyses were conducted using the Nei-Gojobori model in MEGA7.0. All ambiguous positions were removed for each sequence pair. (d) Density of Ks values of Dof orthologous gene pairs between B. napus, B. oleracea, and B. rapa. Analyses were conducted using the Nei-Gojobori model in MEGA7.0. All ambiguous positions were removed for each sequence pair.

Furthermore, we estimated the divergence time of the Dof gene family between Arabidopsis and B. napus by calculating the Ks values of the identified orthologous gene pairs (Table S6a). The Ks value for all the orthologous pairs ranged from 0.28 to 0.74 , with an average of 0.51 (Figure $5 \mathrm{c}$ ). Using the estimate of mutational rate, $\mathrm{R}=1.5 \times 10^{-8}$ synonymous substitutions per site per year [30,31], the average estimated divergence time of the Arabidopsis and B. napus Dof gene family was $\sim 17$ Mya. Our results agree with the reported estimated divergence time (14-24 MYA) of the Arabidopsis and B. napus 
lineage [32,33]. We also calculated the Ks values of orthologous gene pairs between the three Brassica species (Table S6b). The Ks value ranged from 0.0024 to 0.5896 . with an average of 0.15 (Figure $5 \mathrm{~d}$ ). The average divergence time was $\sim 5$ Mya $(80,000$ years$19.5 \mathrm{Mya}$ ). The hybridisation event between $B$. rapa and B. oleracea took place approximately 7500-12,500 years ago, and the Brassica whole-genome triplication event is estimated to have taken place approximately 9-15 Mya $[14,34]$. Overall, these results indicate the divergence of the Brassica genus from Arabidopsis, followed by whole-genome triplication, and hybridisation of B. rapa and B. oleracea to form B. napus, as well as gene loss and rearrangements that shaped the Dof gene family in B. napus.

\subsection{Functional Annotation of BnaDofs and Promoter Analysis}

Functional annotation allows detailed evaluation of proteins with unidentified molecular function, biological processes, or cellular components. In the cellular component gene ontology category, all the BnaDofs were associated with "nucleus", and the majority of them were associated with "integral component of membrane" terms. A Dof gene family is a transcription factor family. It was expected that in the molecular process category, the BnaDofs would be associated with terms such as "DNA binding" and "DNA-binding transcriptional factor activity". In the biological process category, BnaDofs were associated with "regulation of transcription" and several other terms related to organ development, vegetative to reproductive transition, light signalling, response to different hormones, cell differentiation, and oxidation-reduction, among others. A detailed summary of functional annotation results along with descriptors is provided in Table S7.

To gain further understanding of the functional roles of BnaDofs, we used the PlantCARE database to identify potential cis-regulatory elements present upstream of the coding regions (1.5 kb upstream) [35]. Several cis-acting regulatory elements were found in the promoter region of BnaDofs, and we classified them into three categories: developmental, stress-responsive, and hormone-responsive (Figure 6). Among the development-related ciselements, we identified elements regulating light responsiveness (G-box, Box-4, GT1-motif, 3-AF1, AAAC-motif, Sp1, and MRE), circadian rhythm (circadian), meristem expression (CAT-box) and differentiation of palisade mesophyll cells (HD-Zip-1). Cis-acting regulatory elements related to light responsiveness, especially the G-box, Box-4, and GT1-motif elements, were present in $\sim 66 \%, \sim 77 \%$, and $\sim 50 \%$ BnaDofs. CCGTCC-box, a developmentrelated cis-element, was also found in the promoters of 11 BnaDofs, out of which seven BnaDofs belonged to the D major group.

Furthermore, we detected the presence of the stress-responsive cis-elements MBS (involved in drought inducibility), LTR (low-temperature responsive), WUN-motif (wound responsive), TC-rich repeats (defence and stress-related), ARE (anaerobic induction), and GC-motif (anoxic specific inducibility) in 43, 37, 36, 42, 90, and 3 BnaDof promoters, respectively. An as-1 cis-element reported to be present in pathogenesis-related genes in plants was also detected in the promoters of 58 BnaDofs. Stress signalling and hormone signalling operate at an intertwined level in the regulation of plant stress-responsive gene expression. Therefore, we identified hormone-responsive cis-elements in the BnaDof promoters. Among all the hormone-responsive elements, 219 ABRE (Abscisic acid Responsive Elements) elements were present in 77 (68\%) BnaDofs. Cis-elements responsive to auxin (AuxRR-core and TGA-element), gibberellin (TATC-box, GARE-motif, and P-box), salicylic acid (TCA-element), ethylene (ERE), and methyl jasmonate (CGTCA and TGACG) were also present in the BnaDof promoters. In addition to the above-mentioned cis-elements, we encountered several other cis-acting elements such as the AE-box (part of a module for light response), MYB, Myb-binding site, MYC, TATA-box, and CAAT box, which was present in the BnaDof promoters. 


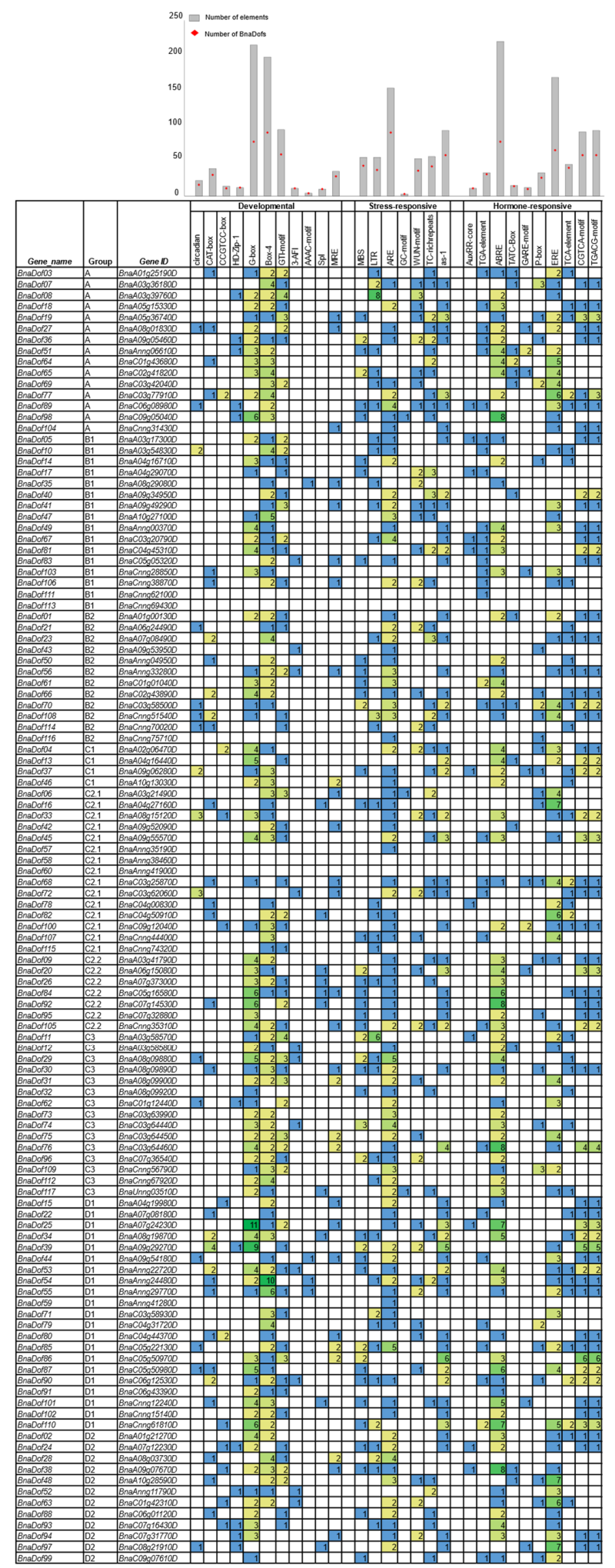

Figure 6. Cis-acting regulatory elements in the promoter region of BnaDof genes. The cis-acting regulatory element analysis in the promoter region ( $1.5 \mathrm{~kb}$ upstream of translation initiation site) of BnaDof genes was performed using the PlantCARE database. The number of each cis-acting element in the promoter regions of BnaDof genes are represented in three major categories: developmental, stress-responsive, and hormone-responsive. On top, the bar graph represents the total number of each cis-acting element present in BnaDofs (grey box) and the corresponding number of BnaDofs promoters carrying a particular cis-element (red diamond). The details of the cis-elements are provided in Table S10. 


\subsection{Tissue-Specific and Abiotic Stress-Responsive Expression Profiling of BnaDofs}

To analyse the tissue-specific expression patterns of BnaDofs, publicly available RNASeq data [14] for four tissues were compared: young root, stem, leaf, and flower buds (Table S10a). The distinctive tissue-specific expression of BnaDofs could be grouped into eight (T.I-T.VIII) clusters, as illustrated in Figure 7a. The majority of the BnaDofs belonging to cluster T.II showed higher expression in young roots. Similarly, T.III and T.VIII showed higher expression in flower buds and stem, respectively. Additionally, BnaDofs belonging to cluster T.I, T.IV, T.V, and T.VII showed higher expression in at least two tissues. Interestingly, in cluster T.VI, BnaDof01, BnaDof34, BnaDof65, and BnaDof101 had higher expression in leaves and flower buds, BnaDof61, BnaDof62, BnaDof66, BnaDof74, and BnaDof116 showed higher expression in leaves, whereas the expression of BnaDof11, BnaDof12, BnaDof29, BnaDof32, BnaDof73, and BnaDof96 was undetectable in any of the four tissues. Preferential expression within the tissue-specific cohort of the Dof family group was also noticeable. For instance, BnaD of members belonging to group C2.1 showed higher expression in young roots and stem; more than $50 \%$ of the A and B1 Dof group members showed preferential expression in stem and young roots, respectively.

a

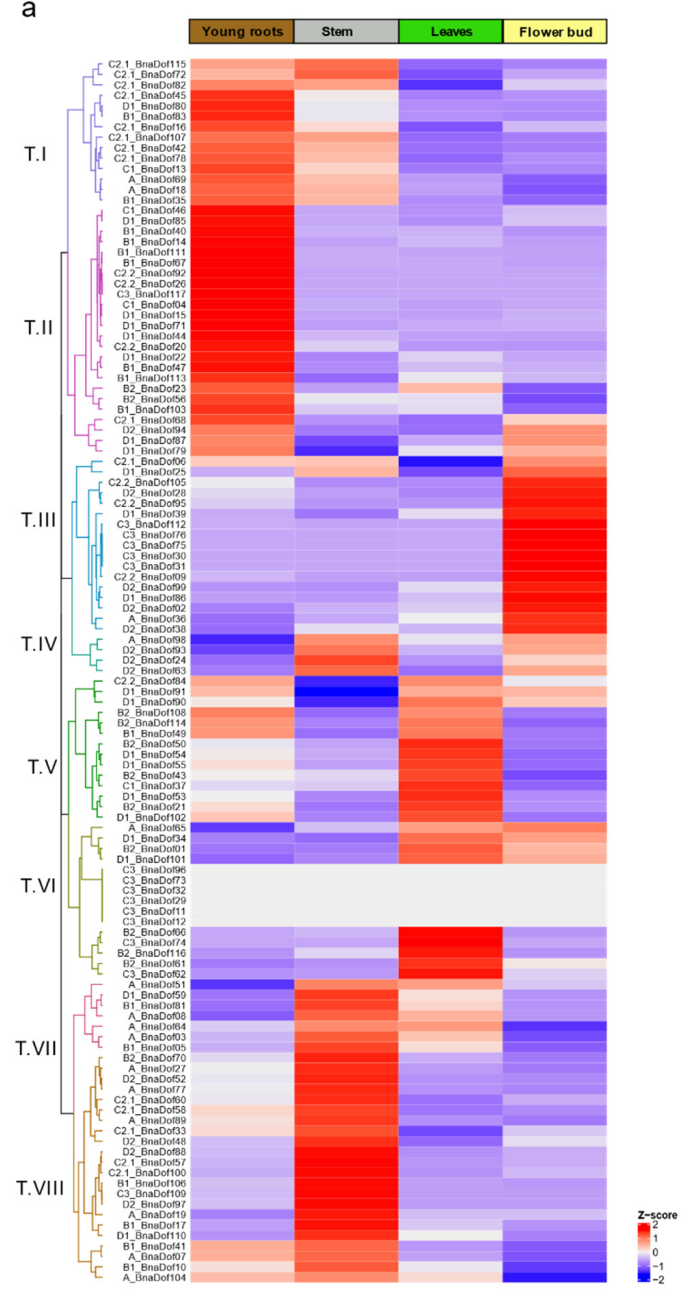

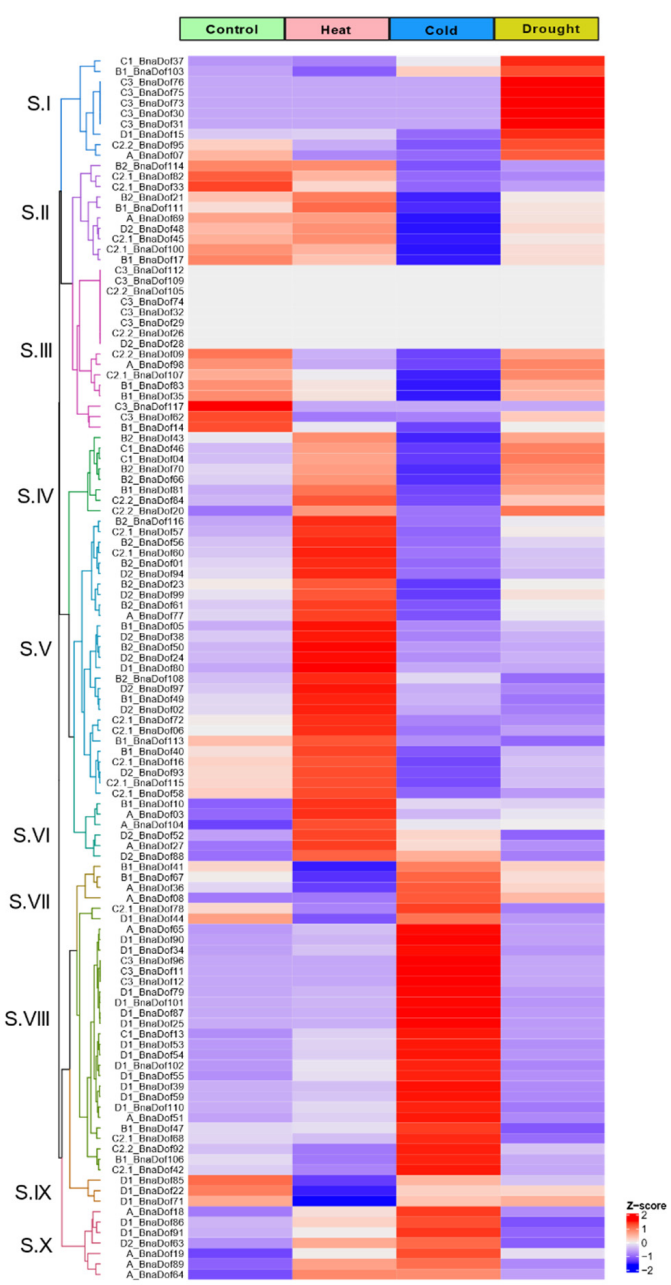

Figure 7. (a) Heat map represents the tissue-specific expression of BnaDofs. RNA-Seq data were obtained from root, stem, leaf, and flower of B. napus. (b) Heat map representation of the stress-responsive expression of BnaDofs. RNA-Seq data were obtained from three weeks old B. napus seedlings grown at control conditions $\left(16 / 8 \mathrm{~h}\right.$ photoperiods at $22{ }^{\circ} \mathrm{C}, \mathrm{RH} 50 \%$, and a light intensity of $230-240 \mu \mathrm{mol} \mathrm{m}{ }^{-2} \mathrm{~s}^{-1}$ ) and exposed to Heat: $35^{\circ} \mathrm{C}$ for $24 \mathrm{~h}$, Cold: $4{ }^{\circ} \mathrm{C}$ for $24 \mathrm{~h}$, and Drought: $22{ }^{\circ} \mathrm{C}$, 25\% PEG-6000 for $24 \mathrm{~h}$. Hierarchical clustering of BnaDof expression profiles was performed using the Euclidean distance method and complete clustering method. The scale bar represents the Z-score (scaled TPM values). 
Brassica Expression DataBase (BrassicaEDB), a gene expression database for Brassica crops (version 1.0) was released recently [36]. To get a comprehensive understanding of developmental expression patterns of BnaDofs, we compiled the expression maps of BnaDofs, across different tissues and developmental stages as available on Brassica EDB (Supplementary Folder). The expression levels of BnaDof11, BnaDof12, BnaDof29, BnaDof32, $B n a D o f 73$, and BnaDof 96 were undetectable in young roots, stem, leaves, and flower buds based on our RNA-Seq analysis (Figure 7a). Thus, we observed the expression maps of these genes on BrassicaEDB. BnaDof73 showed limited expression in cotyledons ( $48 \mathrm{~h}$ after germination), seeds (13 days after fertilisation), and the seed coat (inner integument). Similarly, BnaDof96 and BnaDof11 were only expressed in the seed coat (inner integument), BnaDof32 and BnaDof 29 in seeds 13 days after fertilisation, and BnaDof12 in seeds 10 days after fertilisation.

We further investigated the changes in expression patterns of BnaDofs in three-weekold $B$. napus seedlings exposed to various abiotic stresses by analysing the publicly available RNA-Seq data [36]. Figure 7b illustrates the expression patterns of BnaDofs in control conditions and upon exposure to heat stress, cold stress, and drought conditions (Table S10b). The expression profiles of BnaDofs were grouped into ten clusters (S.1-S.X). Numerous $B n a D$ of genes showed changes in expression profiles upon exposure to extreme temperature. BnaDofs grouped in clusters S.VI, S.VII, S.VIII, and S.X were upregulated in response to low-temperature stress. BnaDof genes belonging to clusters SII, SIII, SIV, SV, and S.IX were downregulated in response to cold stress. The expression of some BnaDofs belonging to cluster S.III (BnaDof 26, BnaDof28, BnaDof29, BnaDof32, BnaDof74, BnaDof105, BnaDof109, and BnaDof112) was undetectable or did not show any changes in expression upon abiotic stress exposure. This probably indicates that these genes might not be involved in heat, cold, and drought stress response in three-week-old seedlings. Cluster S.IV, S.V, and S.VI genes were upregulated upon exposure to heat stress. The majority of the heat-responsive BnaDofs were upregulated. Interestingly a fraction of BnaDofs, which were upregulated in response to heat stress, showed downregulation in response to cold stress and vice versa. For example, BnaDofs clustered in S.IV were upregulated in response to heat and downregulated in response to cold, and an opposite trend was seen in genes clustering in S.VII. Genes belonging to clusters S.I and S.IV showed significant upregulation under drought conditions, and S.II cluster BnaDofs were downregulated. However, the majority of BnaDofs were not drought-responsive.

\section{Discussion}

Brassica napus, the second-largest economically important oilseed crop, is an allopolyploid formed due to the spontaneous pairwise hybridisation of B. rapa and B. oleracea. The availability of the $B$. napus genome provides opportunities for the identification of important gene families. One of the important plant-specific transcription factor families is the Dof zinc finger gene family. This gene family has been described in several plant species, including Arabidopsis [6], rice [7], wheat [8], tomato [37], pepper [38], Chinese cabbage [21], and cucumber [39], among others, but not in B. napus. Functional characterisation of various DOF proteins has highlighted their role in key plant functions such as seed development and germination [40,41], light-regulated hypocotyl elongation [42], photosynthesis [10,43], flowering [44-46], lipid biosynthesis [47,48], carbon metabolism [5], pollen development [49], abiotic stress response [46,50,51], and several other biological processes. In this study, we performed in silico genome-wide identification, comparison, and evolutionary analysis of the Dof gene family in B. napus. Here, we report 117 genes as putative members of the Dof gene family in B. napus, which is the largest number of Dof genes ever reported in eudicots.

\subsection{Systematic Analysis of BnaDofs}

According to the phylogenetic analysis of Dof genes in Arabidopsis and rice, reported by Lijavetzky and collaborators [7], members of the Dof gene family are classified into 
nine groups. The 117 BnaDofs identified in our study were also classified into nine groups based on the phylogenetic analysis between the identified BnaDofs and Arabidopsis Dof genes. We further performed a phylogenetic analysis between Arabidopsis, B. napus, B. rapa, and B. oleracea. Our present study also identified 62 Dof genes in B. oleracea. In B. rapa, 76 Dof genes were reported [21]; however, we included only 74 B. rapa Dof in our analysis. Bra007632 contained a B3 DNA binding domain, auxin response factor domain, Aux/IAA domain, and DOF domain and was orthologous to AUXIN RESPONSE FACTOR 18 Arabidopsis. Bra002057 contained a Syntaxin domain and the DOF domain and was orthologous to a SYNTAXIN OF PLANT 21 gene in Arabidopsis. Thus, these two genes were excluded from our study. The Dof gene family across the Brassica species also clustered phylogenetically in nine groups. Furthermore, comparative analysis of the gene structure and conserved protein domains highlighted conserved exon-intron organisations and distribution of protein motifs followed by most BnaDofs. The DOF domain (motif 1, Figure 3c) was conserved across all BnaDofs, and a similar motif distribution pattern can be seen across the members belonging to the same group. The presence of specific motifs across members of a subgroup, for example, motif 7 being only present in the members of subgroup C3, indicates the specificity of these motifs to the evolution of a subgroup.

The exon-intron organisation of most BnaDofs was similar to Dof genes reported in other plants such as Arabidopsis and rice [7]. The majority of the BnaDof genes had zero to one intron. Eight BnaDofs had two introns, and two BnaDofs (BnaDof 40 and BnaDof86) had three introns. The intron length across members of a subgroup was also diverse. In Arabidopsis, the Dof genes had zero to one intron, and in rice, the Dof genes had zero to two introns [7]. It is worth mentioning that in Arabidopsis, the transcripts/splice variants of Dof genes can have more than one intron; for example, At3g55370.3, a transcript of At3g55370, had three introns. We also observed the exon-intron organisation of $B$. rapa and B. oleracea Dof genes (Figure S2). B. rapa Dof genes had zero to two introns and B. oleracea Dof genes had zero to one intron. The structural diversity of BnaDof genes in terms of intron number suggests that in comparison to the Dof genes of B. rapa and B. oleracea, $B n a D$ ofs acquired introns during evolution. Li et al. 2019 [52] reported that the EIN/EIL3 gene family members in B. napus also acquired introns. The presence of introns can be advantageous for an organism [53]. For instance, due to alternative splicing, the protein diversity of an organism can increase. Introns are also reported to regulate gene expression and produce non-coding RNAs that play diverse regulatory roles.

\subsection{Expansion and Divergence of the Dof Gene Family in B. napus}

Genome-wide studies identifying gene families in $B$. napus have reported the frequent expansion of gene families such as HSF, GST, CRF, TLP, and bHLH, to name a few [54-58]. The difference in the size of the Dof gene family from Arabidopsis to B. napus indicates the expansion of the B. napus Dof gene family. We performed orthologous gene clustering, synteny analysis and molecular evolutionary analysis to understand the expansion of the BnaDofs. The average synonymous base substitution rate between B. napus Dof genes and their Arabidopsis orthologs was calculated as 0.51 (0.28 to 0.74). We further estimated the divergence time of $\sim 17 \mathrm{Mya}$, and it was constant with the time Arabidopsis and Brassica lineages diverged, i.e., 14-24 Mya [33]. Similarly, the calculated maxima, minima, and average Ks values of the orthologous gene pairs between the Brassica species highlights that the whole-genome triplication events (9-15 Mya) and the hybridisation event (7500 years ago) led to the expansion of the Dof gene family in B. napus [14,32]. The expansion of gene families due to whole-genome and local gene duplication events might be an effective strategy in plants for adapting to the ever-changing environmental conditions.

\subsection{Distinct Expression Patterns of BnaDofs during Development}

Tempo-spatial expression profiles of BnaDofs in association with functional annotations and cis-element analysis of BnaDof promoters indicate their preferential expression in different tissues, suggesting a diversification of function during organ development. 
The functional characterisation of DOF proteins in different plants has indicated their association with light-responsiveness, phytochrome signalling, seed germination, and tissue-specific expression in endosperms, vascular tissue development, leaves, or guard cells [11,59-61]. Here in this section, to provide better clarity, we will be discussing the BnaDof genes in terms of orthologous relationships with Arabidopsis Dof genes rather than in terms of classified groups, as different schemes for the Dof gene family classification are available in the literature $[6,7,11]$.

Light is an indispensable environmental cue regulating developmental processes such as photomorphogenesis, seed germination, flowering, and several other metabolic and cellular processes [62]. In plants, the first DOF TF reported in maize was involved in light signalling [3-5]. The BnaDof promoters are enriched for the presence of light-responsive cis-acting elements. Several Dof genes in plants are associated with the regulation of photomorphogenesis, seed germination, and development $[59,60]$.

Light is essential for converting the inactive Pr form of phytochrome into the active Pfr, which then activates the process of seed germination [63]. In Arabidopsis, DAG1, $D A G 2, C O G 1$, and $O B P 3$ were reported to regulate seed germination and hypocotyl elongation [64-67]. DAG1 represses seed germination in response to light, and DAG2 activates seed germination, thus acting antagonistically [64]. In Arabidopsis, the expression of $D A G 1$ and DAG2 is detected in vascular tissues but not in seeds, suggesting regulation of long-distance light-related signalling pathways. The $B$. napus gene orthologous to DAG1 is BnaDof45, and to DAG2 are BnaDof06 and BnaDof68. These genes also showed comparatively higher expression in tissues other than seed or embryo.

The first reported DOF protein to regulate phytochrome-mediated signalling involved in seedling development was COG1 [65]. COG1 interacts with Phytochrome Interacting Factors (PIF4 and PIF5), activates Brassinosteroids biosynthesis, and promotes hypocotyl elongation [68]. It was also reported that COG1 controls the expression of PRX2 and $P R X 25$, which are associated with seed longevity, and thereby regulates seed tolerance [69]. Four BnaDof genes, BnaDof22, BnaDof44, BnaDof71, and BnaDof85, were identified as orthologous to COG1(At1g29160) and functionally annotated to be associated with seed coat development, and showed very high to moderate expression in the seed coat. The Arabidopsis $O B P 3$ gene is involved in hypocotyl elongation repression in a light-dependent manner [67]. Among the orthologs of OBP3, in comparison to BnaDof111, BnaDof40 showed higher expression in hypocotyl, suggesting a similar function.

Circadian rhythms occur in plants to respond to daily and seasonal changes and synchronise their developmental programme based on the day length [70]. In addition to light-responsive cis-elements, a few BnaDof promoters also showed the presence of circadian clock associated cis-elements (Figure 6). In Arabidopsis, cycling DOF factors (CDF1, CDF2, CDF3, and CDF5) were reported to regulate the photoperiodic flowering response [44,71]. In Arabidopsis, CDF1 represses the transcription of a core circadian clock signalling gene CONSTANS (CO) [44,72]. The CO gene is involved in regulating flowering under long days, and its repression by CDF1 represses flowering in Arabidopsis [44,73]. Its ortholog in B. napus, BnCDF1 (BnaDof54 in our study), was also reported to play a role in flowering [74]. BnaDof54, functionally annotated to be involved in flower development, shows very high expression in flowers. Similarly, BnaDof55, which is also orthologous to $C D F 1$, was functionally annotated to be related to flower development and showed higher flower expression, indicating a similar functional role.

The Arabidopsis Dof gene, At3g45610, also known as Dof6 or Dof3.2, negatively regulates seed germination [75]. Orthology analysis revealed the absence of the Dof6 orthologous gene across the B. napus Dof gene family. Gene loss due to the evolution and divergence of BnaDof genes might have either resulted in a loss of function or neofunctionalisation. It is worth mentioning that the cis-element analysis of the BnaDof promoters also revealed cis-elements related to or acting as binding sites for other transcription factors (MYB, MYC, ARF, and MADS-boxes). A similar diversification of binding sites was 
reported for Arabidopsis Dof promoters, indicating potential relationships between DOF TFs and other TFs regulating diverse plant developmental processes [76].

In cereals, Dof genes play a role in seed protein accumulation and mobilisation [60,77,78]. In maize, ZmDof36 and ZmDof3 play a role in seed starch accumulation $[79,80]$. However, for B. napus, the accumulation of oil in the seeds is of economic importance. In soybean, two DOF-like proteins, GmDOF4 and GmDOF11, enhance the fatty acid content in seeds when overexpressed in Arabidopsis by directly binding to the promoter regions of the acetyl CoA carboxylase gene and long-chain-acyl CoA synthetase gene and activating their expression [47]. Similarly, the overexpression of the GhDof1 gene in cotton can potentially increase seed lipid content [48]. Thus, further exploring the role of BnaDof genes in regulating seed oil content will prove beneficial in producing high oil yielding varieties.

\subsection{Potential Role of BnaDofs in Abiotic Stress Response}

DOF TFs have also been reported to participate in response to the abiotic stress response [62]. Genome-wide expression analysis studies have reported the abiotic stressresponsive gene expression of Dof genes in Chinese cabbage [21], wheat [8], tomato [45], pepper [38], rose [81], and other plant species. We investigated the expression profiles of BnaDofs in response to heat, cold, and drought stress. In response to heat stress, the majority of the differentially regulated BnaDofs were upregulated. The mechanism by which Dof genes regulate heat stress response is not yet described for Dof genes. In walnut, Yang et al. [82] suggested the contribution of $J r D o f 3$ in enhancing the heat stress response of JrGRAS2. JrGRAS2 overexpression lines in Arabidopsis exhibited enhanced heat stress tolerance.

The majority of BnaDofs in our analysis were differentially regulated upon exposure to low temperatures. A recent study in B. napus exploring the cold-responsive TFs reported the changes in expression of Dof genes in response to the cold stress response and suggested their possible role in imparting cold tolerance [50]. In cotton, the overexpression of the GhDof1 gene enhanced cold tolerance during the seedling stage [48]. The transgenic line overexpressing GhDof1 also exhibited enhanced salinity tolerance due to enhanced root development in transgenics under salt stress.

In B. napus, BnCDF1 (BnaDof54 in our study) has been reported to play a role in freezing tolerance [74]. Based on our expression analysis, this gene was highly upregulated upon exposure to $4{ }^{\circ} \mathrm{C}$ for $24 \mathrm{~h}$. Furthermore, the overexpression of two tomato $C D F$ genes (SlCDF1 and SlCDF3) in Arabidopsis enhanced drought and salt tolerance [45]. The overexpression of Arabidopsis CDF3 also enhanced the tolerance of transgenic Arabidopsis plants to drought, cold, and osmotic stress [46]. A tomato transgenic line overexpressing $A t C D F 3$ and SlCDF3 exhibited an enhanced growth rate and yield under control and salt stress conditions [83]. Transcriptomic analysis of these transgenic tomato lines revealed the role of CDF3 in regulating the expression of several genes involved in cell growth, metabolism, and stress response. The CDF3 orthologous genes in B. napus, BnaDof53 and $B n a D o f 102$, significantly upregulated in response to cold stress and slightly in response to heat stress. In comparison to control conditions, these two genes showed a slight reduction in gene expression under drought. CDFs thus play a potential role in abiotic stress tolerance, in addition to their role in flowering time control.

We further observed a few BnaDofs $(-17,-47,-81$, and -113) associated with the functional terms "oxidation-reduction process" and "response to oxidative stress", suggesting a potential role in ROS-mediated signalling. In wheat, some TaDofs have been suggested to act as dynamic regulators of ROS clearance pathways based on their response to heavy metal stress [8]. DOF proteins are also involved in phytohormone signalling pathways [84]. Our cis-acting element analysis of the BnaDof promoters and the functional annotation of BnaDof proteins revealed the presence of several cis-elements responsive to auxin, abscisic acid, salicylic acid, gibberellic acid, and methyl jasmonate. ABRE elements were enriched in the promoters of 77 BnaDofs. ABA-dependent pathways and phytohormone signalling 
are known to respond to abiotic stresses $[85,86]$. This suggests that hormones can activate BnaDof expression, and they might play a role in stress signalling pathways.

The expression analysis exemplifies the stress-responsive nature of BnaDofs. The differential regulation of BnaDofs may regulate downstream genes involved in stress response, probably imparting tolerance. Different BnaDofs can be stress-responsive in different tissues and at different developmental stages, due to the preferential tissue expression of BnaDofs. It is also important to note that depending upon the variety and even plant species, the expression profiles and functional roles of Dof genes in response to stress may show variation. Overall, this study provides a comprehensive understanding of the molecular structure, evolution, and potential functions of BnaDofs.

\section{Materials and Methods}

\subsection{Identification of Dof Gene Family Members in B. napus}

A BLASTP search of the B. napus proteome was carried out using zf-DOF-domain search model accession (Pfam: PF02701) as a query to obtain the consensus aminoacid sequences of the putative DOF proteins. The term 'Dof' and DOF-domain search model accession 'PF02701' were used to search the Plant Transcription Factor Database 4.0 database (PlantTFDB) [18]. To identify the integrated DOF domain in the putative DOFs obtained from the BLASTP and PlantTFDb search, SMART 8.0 software ( http://smart.embl-heidelberg.de/, accessed on 5 August 2020) was used, and the final predicted DOFs were further characterised [19]. Expasy server's ProtParam tool ( https:/ /web.expasy.org/protparam/, accessed on 7 August 2020) was used to compute the various physical and chemical properties of the predicted Dof proteins such as the number of amino acids in the protein sequence, molecular weight $(\mathrm{Mw})$, protein isoelectric point (pI), and Grand Average of Hydropathy (GRAVY) of the protein [87]. Chromosomal locations as well as the genomic, coding, peptide, and promoter sequences of the DOF TFs were downloaded from GenoScope (Brassica napus. annotation_v5) database. Unique gene identifiers were assigned to the DOFs, and they were referred to as BnaDofs.

\subsection{Evolutionary and Gene Duplication Analysis of BnaDofs}

Multiple sequence alignments were performed on the DOF amino acid sequences using CLUSTALW with default settings [23]. MEGA7.0 was used to construct a phylogenetic tree based on the maximum likelihood method based on the JTT matrix-based method. Statistical support for each tree node was provided by performing a 100-500 replicate bootstrap analysis [24]. We also constructed the phylogenetic tree of BnaDofs using the neighbour-joining (NJ) method with 1000 bootstrap replicates, and the Poisson correction method.

To identify gene duplications in BnaDofs, all B. napus gene sequences (101040) were first aligned using BLASTp, with an e-value of 1e-10, and then the duplication patterns were classified into interspersed and tandem duplications with MCScanX (default parameters) [20,27]. Gene duplication was also analysed based on sequence similarity criteria, i.e., the similarity of the aligned regions of protein $\geq 80 \%$ [40]. Evolutionary analyses were conducted in MEGA7 [24]. The number of synonymous substitutions per synonymous site $(\mathrm{dS} / \mathrm{Ks})$, and the number of non-synonymous substitutions per non-synonymous site $(\mathrm{dN} / \mathrm{Ka})$, were calculated using the Nei-Gojobori method (Jukes-Cantor). The formula $\mathrm{T}=\mathrm{Ks} / 2 \mathrm{R}$ (where, $\mathrm{Ks}=$ number of synonymous substitutions per synonymous site, $\mathrm{R}=1.5 \times 10^{-8}$ synonymous substitutions per site per year, and $\mathrm{T}=$ divergence time) was used to estimate divergence time $[33,88]$.

\subsection{Gene Structure and Motif Analysis of BnaDofs}

The gene structure in terms of the exon-intron organisation was determined using the GSDS2.0 (Gene Structure Display Server; http://gsds.cbi.pku.edu.cn, accessed on 23 September 2020) [73,89]. The MEME tool from the MEME suite 5.1.1 (http://memesuite.org/tools/meme) was used to identify fifteen statistically significant motifs of the 
BnaDof protein sequences based on "zero or one occurrence per sequence (zoops)" [25]. The discoverable motif length and sites were set to 6-50 and 2-600, respectively.

\subsection{Functional Annotation and Promoter Analysis}

Functional annotation of BnaDofs was performed using PANNZER2 (Protein ANNotation with Z-scoRE 2), which provided both Gene Ontology (GO) annotations and free text description predictions [90]. Promoter analysis of the BnaDof genes was performed by using the PlantCARE database (http:/ / bioinformatics.psb.ugent.be/webtools/plantcare/html/, accessed on 25 September 2020) to identify the cis-acting regulatory elements with putative involvement in various abiotic stress responses [35]. $1500 \mathrm{bp}$ upstream regions from the start codon (ATG) of the Dof genes were downloaded as the promoter sequences from the EnsemblPlants Database [91].

\subsection{Orthology and Collinearity Analysis of BnaDofs}

Genome annotations and peptide sequences were downloaded from GenoScope (Brassica_napus.annotation_v5) and EnsemblPlants (Brassica_rapa.IVFCAASv1.36, Brassica_oleracea.v2.1.36). The orthologous genes in B. oleracea, B. rapa, B. napus, and A. thaliana were identified using OrthoVenn2 (https: / / orthovenn2.bioinfotoolkits.net/home, accessed on 27 September 2020) [26].

For the analysis of collinearity/synteny, 58 out of 117 DOF transcription factor members identified in B. napus were discarded because their chromosome of origin was uncertain. Syntenic blocks were identified by a sequence similarity search of the remaining 59 DOF members in B. napus against the reference genomes of Arabidopsis, B. rapa, and B. oleracea, using Blastn v. 2.10.1+ with stringent parameters (cut-off e-value 10e-50nd a minimum percentage of identity of 75) [20]. A custom python script was used to reformat the blast output and identify collinear genes and genes that underwent chromosomal translocation. Synteny plots were plotted with CIRCOS v. 0.69-9 [92].

\subsection{Tissue-Specific Expression and Abiotic Stress Response Expression Profiling of BnaDofs}

To investigate tissue-specific expression (under non-stressed conditions) and abiotic stress-responsive expression of the BnaDof genes, RNA-Seq data sets from previously published literature $[14,36]$ were downloaded from the NCBI Sequence Read Archive database (Table S11). Transcript expression was quantified using Kallisto v0.44.0, and read abundance was expressed as Transcripts Per Kilobase Million (TPM) [93]. Heat maps were drawn by using the ComplexHeatmap package to visualise the expression of BnaDofs [94]. Additionally, the expression maps for BnaDofs were downloaded from the Brassica expression DataBase (BrassicaEDB). The Supplementary Folder with the downloaded expression maps of BnaDofs is accessible via the following link https://jmp. sh/odBHlfT.

\section{Conclusions}

A systematic analysis of the B. napus Dof transcription factor gene family identified a total of 117 BnaDofs. The BnaDofs were classified into nine groups: A, B1, B2, C1, C2.1, C2.2, C3, D1, and D2 based on the phylogenetic analysis. Based on the orthology, synteny, and evolutionary analysis, the calculated divergence times indicated that the divergence of the Brassica and Arabidopsis genus ( 17 Mya), the whole-genome triplication event (9-15 Mya), and the formation of B. napus (7500 years ago) drove the expansion of the BnaDof gene family. Synteny analysis also highlighted that the majority of the Dof genes with known chromosomal locations in B. napus did not undergo translocations. The $\mathrm{Ka} / \mathrm{Ks}$ ratio of the duplicated gene pairs indicated that the BnaDof gene pairs underwent purifying selection. Further understanding of the molecular evolutionary mechanism is required to understand how gene duplications, gene loss, and rearrangements can lead to the expansion of gene families and the possible neo- or sub- functionalisation of genes. Tissue-specific expression highlighted the role of BnaDofs in organ development 
and other developmental processes. Most of the BnaDofs were responsive to temperature fluctuations and were differentially regulated, particularly by cold stress. Additionally, molecular characterisation, functional annotation, and cis-acting element analysis have provided a starting point for further research investigations-our study supports the involvement of the Dof gene family in developmental processes and multiple abiotic stress responses. Further research is warranted to dissect the role of BnaDofs and explore these transcriptional regulators for developing climate change-resilient varieties with desirable physiological and agronomic traits.

Supplementary Materials: The following are available online at https:/ / www.mdpi.com/article/10 .3390/plants10040709/s1: Figure S1. Phylogenetic tree of Arabidopsis, B. napus, B. oleracea, and B. rapa DOF proteins. The evolutionary history was inferred by using the maximum likelihood method based on the JTT matrix-based method. Maximum-likelihood bootstrap values (100 replicates) above $70 \%$ are shown. The analysis involved 289 amino acid sequences. There was a total of 651 positions in the final dataset. Phylogenetic topology was generated via MEGA7. Figure S2. Gene structure of (a) B. rapa Dof genes, (b) B. oleracea Dof genes. Table S1. Information of BnaDofs, including gene ID, type of Dof, chromosomal location, protein sequence length, isoelectric point ( $\mathrm{pI}$ ), molecular weight (Mw), instability index (I.I), stability, aliphatic index (A.I), and grand average of hydropathicity (GRAVY). Table S2. Summary of Dof genes identified in B. oleracea and reported Dof genes in B. rapa and Arabidopsis. Table S3. Detailed summary of identified syntenic genes between A sub-genome of B. napus and B. rapa, and C sub-genome of B. napus and B. oleracea. Table S4a. Detailed list of orthologous gene clusters between the Dof genes in B. rapa, B. oleracea, B. napus, and Arabidopsis. Table S4b. Detailed list of singleton Dof genes in Arabidopsis, B. napus, and B. rapa. Table S5a. Detailed summary of gene duplications in BnaDofs based on similarity criteria ( $\geq 80 \%)$. Table S5b. Detailed summary of gene duplications along with type of duplications in BnaDofs as predicted by MCScanX. Table S6a. Orthologous gene pairs of Arabidopsis and B. napus Dof genes and their respective Ks values. Table S6b. Orthologous gene pairs of Dof genes across the three Brassica species (B. napus, B. oleracea, and B. rapa) and their respective Ks values. Table S7. Detailed results of functional annotation of BnaDof proteins based on PANNZER2 output. Table S8. Cis-acting regulatory elements detected in the BnaDof promoters. Table S9. Description of cis-acting regulatory elements detected in the BnaDof promoters (used in Figure 6). Table S10a. Tissue-specific RNA-Seq expression data. Read abundance is showed in terms of Transcripts Per Kilobase Million (TPM). Table S10b. RNA-Seq expression data for three-week-old B. napus seedlings grown in control conditions and exposed to different abiotic stresses. Read abundance is showed in terms of Transcripts Per Kilobase Million (TPM). Table S11. Summary of the sequencing data used for analysis. Supplementary Folder: Expression maps of BnaDofs (https://jmp.sh/odBHlfT).

Author Contributions: Conceptualisation, N.L., M.B.S. and P.L.B.; methodology, N.L.; software, N.L.; validation, N.L.; formal analysis, N.L.; investigation, N.L.; resources, N.L. and S.B.; data curation, N.L. and S.B.; writing—original draft preparation, N.L.; writing—review and editing, N.L., S.B., M.B.S. and P.L.B.; visualisation, N.L. and S.B.; supervision, P.L.B. and M.B.S.; project administration, P.L.B. All authors have read and agreed to the published version of the manuscript.

Funding: ARC Discovery grant DP0988972 and the University of Melbourne Research Scholarship.

Institutional Review Board Statement: Not applicable.

Informed Consent Statement: Not applicable.

Data Availability Statement: All data generated or analysed during this study are included in this article and its supplementary information files. Details and accession numbers of the RNA-Seq data libraries downloaded from NCBI sequence Read Archive are outlined in Table S9. The expression maps of BnaDofs downloaded from the Brassica Expression DataBase (BrassicaEDB) are accessible via the following link https://jmp.sh/odBHlfT.

Acknowledgments: This research was supported by Melbourne Bioinformatics at the University of Melbourne, project UOM0033. We also acknowledge the support of the ARC Discovery grant DP0988972 and the University of Melbourne Research Scholarship.

Conflicts of Interest: The authors declare no conflict of interest. 


\section{References}

1. USDA, F.A.S. Oilseeds: World Markets and Trade; USDA Foreign Agricultural Service: Wasington, DC, USA, 2020.

2. Lohani, N.; Jain, D.; Singh, M.B.; Bhalla, P.L. Engineering Multiple Abiotic Stress Tolerance in Canola, Brassica napus. Front. Plant Sci. 2020, 11. [CrossRef]

3. Yanagisawa, S. A novel DNA-binding domain that may form a single zinc finger motif. Nucleic Acids Res. 1995, 23, 3403-3410. [CrossRef]

4. Yanagisawa, S.; Sheen, J. Involvement of maize Dof zinc finger proteins in tissue-specific and light-regulated gene expression. Plant Cell 1998, 10, 75-89. [CrossRef]

5. Yanagisawa, S. Dof1 and Dof2 transcription factors are associated with expression of multiple genes involved in carbon metabolism in maize. Plant J. 2000, 21, 281-288. [CrossRef] [PubMed]

6. Yanagisawa, S. The Dof family of plant transcription factors. Trends Plant Sci. 2002, 7, 555-560. [CrossRef]

7. Lijavetzky, D.; Carbonero, P.; Vicente-Carbajosa, J. Genome-wide comparative phylogenetic analysis of the rice and Arabidopsis Dof gene families. BMC Evol. Biol. 2003, 3, 17. [CrossRef] [PubMed]

8. Liu, Y.; Liu, N.; Deng, X.; Liu, D.; Li, M.; Cui, D.; Hu, Y.; Yan, Y. Genome-wide analysis of wheat DNA-binding with one finger (Dof) transcription factor genes: Evolutionary characteristics and diverse abiotic stress responses. BMC Genom. 2020, 21, 1-18. [CrossRef]

9. Tamai, H.; Iwabuchi, M.; Meshi, T. Arabidopsis GARP transcriptional activators interact with the Pro-rich activation domain shared by G-box-binding bZIP factors. Plant Cell Physiol. 2002, 43, 99-107. [CrossRef]

10. Yanagisawa, S. Dof domain proteins: Plant-specific transcription factors associated with diverse phenomena unique to plants. Plant Cell Physiol. 2004, 45, 386-391. [CrossRef]

11. Le Hir, R.; Bellini, C. The plant-specific dof transcription factors family: New players involved in vascular system development and functioning in Arabidopsis. Front. Plant Sci. 2013, 4, 164. [CrossRef] [PubMed]

12. Umemura, Y.; Ishiduka, T.; Yamamoto, R.; Esaka, M. The Dof domain, a zinc finger DNA-binding domain conserved only in higher plants, truly functions as a Cys2/Cys2 Zn finger domain. Plant J. 2004, 37, 741-749. [CrossRef]

13. Krebs, J.; Mueller-Roeber, B.; Ruzicic, S. A novel bipartite nuclear localization signal with an atypically long linker in DOF transcription factors. J. Plant Physiol. 2010, 167, 583-586. [CrossRef]

14. Chalhoub, B.; Denoeud, F.; Liu, S.; Parkin, I.A.; Tang, H.; Wang, X.; Chiquet, J.; Belcram, H.; Tong, C.; Samans, B. Early allopolyploid evolution in the post-Neolithic Brassica napus oilseed genome. Science 2014, 345, 950-953. [CrossRef]

15. Cheng, F.; Wu, J.; Cai, X.; Liang, J.; Freeling, M.; Wang, X. Gene retention, fractionation and subgenome differences in polyploid plants. Nat. Plants 2018, 4, 258-268. [CrossRef] [PubMed]

16. Liu, S.; Liu, Y.; Yang, X.; Tong, C.; Edwards, D.; Parkin, I.A.; Zhao, M.; Ma, J.; Yu, J.; Huang, S. The Brassica oleracea genome reveals the asymmetrical evolution of polyploid genomes. Nat. Commun. 2014, 5, 1-11. [CrossRef]

17. Wang, X.; Wang, H.; Wang, J.; Sun, R.; Wu, J.; Liu, S.; Bai, Y.; Mun, J.-H.; Bancroft, I.; Cheng, F. The genome of the mesopolyploid crop species Brassica rapa. Nat. Genet. 2011, 43, 1035-1039. [CrossRef] [PubMed]

18. Jin, J.; Tian, F.; Yang, D.-C.; Meng, Y.-Q.; Kong, L.; Luo, J.; Gao, G. PlantTFDB 4.0: Toward a central hub for transcription factors and regulatory interactions in plants. Nucleic Acids Res. 2016, gkw982. [CrossRef]

19. Letunic, I.; Bork, P. 20 years of the SMART protein domain annotation resource. Nucleic Acids Res. 2018, 46, D493-D496. [CrossRef] [PubMed]

20. Korf, I.; Yandell, M.; Bedell, J. Blast; O’Reilly Media Inc., 2003. Available online: https://www.oreilly.com/library/view/blast/05 96002998/ (accessed on 7 April 2021).

21. Ma, J.; Li, M.-Y.; Wang, F.; Tang, J.; Xiong, A.-S. Genome-wide analysis of Dof family transcription factors and their responses to abiotic stresses in Chinese cabbage. BMC Genom. 2015, 16, 1-15. [CrossRef] [PubMed]

22. Lipka, V.; Kwon, C.; Panstruga, R. SNARE-ware: The role of SNARE-domain proteins in plant biology. Annu. Rev. Cell Dev. Biol. 2007, 23, 147-174. [CrossRef]

23. Larkin, M.A.; Blackshields, G.; Brown, N.P.; Chenna, R.; McGettigan, P.A.; McWilliam, H.; Valentin, F.; Wallace, I.M.; Wilm, A.; Lopez, R. Clustal W and Clustal X version 2.0. Bioinformatics 2007, 23, 2947-2948. [CrossRef]

24. Kumar, S.; Nei, M.; Dudley, J.; Tamura, K. MEGA: A biologist-centric software for evolutionary analysis of DNA and protein sequences. Brief. Bioinform. 2008, 9, 299-306. [CrossRef]

25. Bailey, T.L.; Johnson, J.; Grant, C.E.; Noble, W.S. The MEME suite. Nucleic Acids Res. 2015, 43, W39-W49. [CrossRef] [PubMed]

26. Xu, L.; Dong, Z.; Fang, L.; Luo, Y.; Wei, Z.; Guo, H.; Zhang, G.; Gu, Y.Q.; Coleman-Derr, D.; Xia, Q. OrthoVenn2: A web server for whole-genome comparison and annotation of orthologous clusters across multiple species. Nucleic Acids Res. 2019, 47, W52-W58. [CrossRef]

27. Wang, Y.; Tang, H.; DeBarry, J.D.; Tan, X.; Li, J.; Wang, X.; Lee, T.-h.; Jin, H.; Marler, B.; Guo, H. MCScanX: A toolkit for detection and evolutionary analysis of gene synteny and collinearity. Nucleic Acids Res. 2012, 40, e49. [CrossRef]

28. Yang, Z.; Bielawski, J.P. Statistical methods for detecting molecular adaptation. Trends Ecol. Evol. 2000, 15, 496-503. [CrossRef]

29. Li, J.; Zhang, Z.; Vang, S.; Yu, J.; Wong, G.K.-S.; Wang, J. Correlation between Ka/Ks and Ks is related to substitution model and evolutionary lineage. J. Mol. Evol. 2009, 68, 414-423. [CrossRef]

30. Koch, M.; Haubold, B.; Mitchell-Olds, T. Molecular systematics of the Brassicaceae: Evidence from coding plastidic matK and nuclear Chs sequences. Am. J. Bot. 2001, 88, 534-544. [CrossRef] 
31. Koch, M.; Bishop, J.; Mitchell-Olds, T.; Koch, M. Molecular systematics and evolution of Arabidopsis and Arabis. Plant Biol. 1999, 1, 529-537. [CrossRef]

32. Cheung, F.; Trick, M.; Drou, N.; Lim, Y.P.; Park, J.-Y.; Kwon, S.-J.; Kim, J.-A.; Scott, R.; Pires, J.C.; Paterson, A.H. Comparative analysis between homoeologous genome segments of Brassica napus and its progenitor species reveals extensive sequence-level divergence. Plant Cell 2009, 21, 1912-1928. [CrossRef]

33. Koch, M.A.; Haubold, B.; Mitchell-Olds, T. Comparative evolutionary analysis of chalcone synthase and alcohol dehydrogenase loci in Arabidopsis, Arabis, and related genera (Brassicaceae). Mol. Biol. Evol. 2000, 17, 1483-1498. [CrossRef]

34. Cheng, F.; Wu, J.; Wang, X. Genome triplication drove the diversification of Brassica plants. Hortic. Res. 2014, 1, 1-8. [CrossRef]

35. Lescot, M.; Déhais, P.; Thijs, G.; Marchal, K.; Moreau, Y.; Van de Peer, Y.; Rouzé, P.; Rombauts, S. PlantCARE, a database of plant cis-acting regulatory elements and a portal to tools for in silico analysis of promoter sequences. Nucleic Acids Res. 2002, 30, 325-327. [CrossRef] [PubMed]

36. Lee, J.S.; Adams, K.L. Global insights into duplicated gene expression and alternative splicing in polyploid Brassica napus under heat, cold, and drought stress. Plant Genome 2020, 13, e20057. [CrossRef]

37. Cai, X.; Zhang, Y.; Zhang, C.; Zhang, T.; Hu, T.; Ye, J.; Zhang, J.; Wang, T.; Li, H.; Ye, Z. Genome-Wide analysis of plant-Specific Dof transcription factor family in tomato. J. Integr. Plant Biol. 2013, 55, 552-566. [CrossRef]

38. Wu, Z.; Cheng, J.; Cui, J.; Xu, X.; Liang, G.; Luo, X.; Chen, X.; Tang, X.; Hu, K.; Qin, C. Genome-wide identification and expression profile of Dof transcription factor gene family in pepper (Capsicum annuum L.). Front. Plant Sci. 2016, 7, 574. [CrossRef] [PubMed]

39. Wen, C.-1.; Cheng, Q.; Zhao, L.; Mao, A.; Yang, J.; Yu, S.; Weng, Y.; Xu, Y. Identification and characterisation of Dof transcription factors in the cucumber genome. Sci. Rep. 2016, 6, 23072. [CrossRef] [PubMed]

40. Gabriele, S.; Rizza, A.; Martone, J.; Circelli, P.; Costantino, P.; Vittorioso, P. The Dof protein DAG1 mediates PIL5 activity on seed germination by negatively regulating GA biosynthetic gene AtGA3ox1. Plant J. 2010, 61, 312-323. [CrossRef]

41. Santopolo, S.; Boccaccini, A.; Lorrai, R.; Ruta, V.; Capauto, D.; Minutello, E.; Serino, G.; Costantino, P.; Vittorioso, P. DOF AFFECTING GERMINATION 2 is a positive regulator of light-mediated seed germination and is repressed by DOF AFFECTING GERMINATION 1. BMC Plant Biol. 2015, 15, 1-11. [CrossRef]

42. Martín, G.; Veciana, N.; Boix, M.; Rovira, A.; Henriques, R.; Monte, E. The photoperiodic response of hypocotyl elongation involves regulation of CDF1 and CDF5 activity. Physiol. Plant. 2020, 169, 480-490. [CrossRef]

43. Shaw, L.M.; McIntyre, C.L.; Gresshoff, P.M.; Xue, G.-P. Members of the Dof transcription factor family in Triticum aestivum are associated with light-mediated gene regulation. Funct. Integr. Genom. 2009, 9, 485-498. [CrossRef]

44. Fornara, F.; Panigrahi, K.C.; Gissot, L.; Sauerbrunn, N.; Rühl, M.; Jarillo, J.A.; Coupland, G. Arabidopsis DOF transcription factors act redundantly to reduce CONSTANS expression and are essential for a photoperiodic flowering response. Dev. Cell 2009, 17, 75-86. [CrossRef]

45. Corrales, A.-R.; Nebauer, S.G.; Carrillo, L.; Fernández-Nohales, P.; Marqués, J.; Renau-Morata, B.; Granell, A.; Pollmann, S.; Vicente-Carbajosa, J.; Molina, R.-V. Characterization of tomato Cycling Dof Factors reveals conserved and new functions in the control of flowering time and abiotic stress responses. J. Exp. Bot. 2014, 65, 995-1012. [CrossRef]

46. Corrales, A.R.; Carrillo, L.; Lasierra, P.; Nebauer, S.G.; Dominguez-Figueroa, J.; Renau-Morata, B.; Pollmann, S.; Granell, A.; Molina, R.V.; Vicente-Carbajosa, J. Multifaceted role of cycling DOF factor 3 (CDF3) in the regulation of flowering time and abiotic stress responses in Arabidopsis. PlantCell Environ. 2017, 40, 748-764. [CrossRef]

47. Wang, H.W.; Zhang, B.; Hao, Y.J.; Huang, J.; Tian, A.G.; Liao, Y.; Zhang, J.S.; Chen, S.Y. The soybean Dof-type transcription factor genes, GmDof4 and GmDof11, enhance lipid content in the seeds of transgenic Arabidopsis plants. Plant J. 2007, 52, 716-729. [CrossRef] [PubMed]

48. Su, Y.; Liang, W.; Liu, Z.; Wang, Y.; Zhao, Y.; Ijaz, B.; Hua, J. Overexpression of GhDof1 improved salt and cold tolerance and seed oil content in Gossypium hirsutum. J. Plant Physiol. 2017, 218, 222-234. [CrossRef] [PubMed]

49. Chen, X.; Wang, D.; Liu, C.; Wang, M.; Wang, T.; Zhao, Q.; Yu, J. Maize transcription factor Zmdof1 involves in the regulation of Zm401 gene. Plant Growth Regul. 2012, 66, 271-284. [CrossRef]

50. Ke, L.; Lei, W.; Yang, W.; Wang, J.; Gao, J.; Cheng, J.; Sun, Y.; Fan, Z.; Yu, D. Genome-wide identification of cold responsive transcription factors in Brassica napus L. BMC Plant Biol. 2020, 20, 62. [CrossRef]

51. Renau-Morata, B.; Carrillo, L.; Dominguez-Figueroa, J.; Vicente-Carbajosa, J.; Molina, R.V.; Nebauer, S.G.; Medina, J. CDF transcription factors: Plant regulators to deal with extreme environmental conditions. J. Exp. Bot. 2020. [CrossRef] [PubMed]

52. Li, M.; Wang, R.; Liang, Z.; Wu, X.; Wang, J. Genome-wide identification and analysis of the EIN3/EIL gene family in allotetraploid Brassica napus reveal its potential advantages during polyploidization. Bmc Plant Biol. 2019, 19, 1-16. [CrossRef] [PubMed]

53. Jo, B.-S.; Choi, S.S. Introns: The functional benefits of introns in genomes. Genom. Inform. 2015, 13, 112. [CrossRef]

54. Lohani, N.; Golicz, A.A.; Singh, M.B.; Bhalla, P.L. Genome-wide analysis of the Hsf gene family in Brassica oleracea and a comparative analysis of the Hsf gene family in B. oleracea, B. rapa and B. napus. Funct. Integr. Genom. 2019, 19, 515-531. [CrossRef]

55. Wei, L.; Zhu, Y.; Liu, R.; Zhang, A.; Zhu, M.; Xu, W.; Lin, A.; Lu, K.; Li, J. Genome wide identification and comparative analysis of glutathione transferases (GST) family genes in Brassica napus. Sci. Rep. 2019, 9, 1-13. [CrossRef]

56. Wang, S.; Zhang, H.; Shi, L.; Xu, F.; Ding, G. Genome-Wide Dissection of the CRF Gene Family in Brassica napus Indicates that BnaCRF8s Specifically Regulate Root Architecture and Phosphate Homeostasis against Phosphate Fluctuation in Plants. Int. J. Mol. Sci. 2020, 21, 3660. [CrossRef] [PubMed] 
57. Wang, T.; Hu, J.; Ma, X.; Li, C.; Yang, Q.; Feng, S.; Li, M.; Li, N.; Song, X. Identification, evolution and expression analyses of whole genome-wide TLP gene family in Brassica napus. BMC Genom. 2020, 21, 1-14. [CrossRef]

58. Miao, L.; Gao, Y.; Zhao, K.; Kong, L.; Yu, S.; Li, R.; Liu, K.; Yu, X. Comparative analysis of basic helix-loop-helix gene family among Brassica oleracea, Brassica rapa, and Brassica napus. BMC Genom. 2020, 21, 1-18. [CrossRef]

59. Noguero, M.; Atif, R.M.; Ochatt, S.; Thompson, R.D. The role of the DNA-binding One Zinc Finger (DOF) transcription factor family in plants. Plant Sci. 2013, 209, 32-45. [CrossRef]

60. Ruta, V.; Longo, C.; Lepri, A.; De Angelis, V.; Occhigrossi, S.; Costantino, P.; Vittorioso, P. The DOF Transcription Factors in Seed and Seedling Development. Plants 2020, 9, 218. [CrossRef]

61. Moreno-Risueno, M.Á.; Díaz, I.; Carrillo, L.; Fuentes, R.; Carbonero, P. The HvDOF19 transcription factor mediates the abscisic acid-dependent repression of hydrolase genes in germinating barley aleurone. Plant J. 2007, 51, 352-365. [CrossRef]

62. Legris, M.; Nieto, C.; Sellaro, R.; Prat, S.; Casal, J.J. Perception and signalling of light and temperature cues in plants. Plant J. 2017, 90, 683-697. [CrossRef]

63. Shinomura, T.; Nagatani, A.; Chory, J.; Furuya, M. The induction of seed germination in Arabidopsis thaliana is regulated principally by phytochrome B and secondarily by phytochrome A. Plant Physiol. 1994, 104, 363-371. [CrossRef]

64. Gualberti, G.; Papi, M.; Bellucci, L.; Ricci, I.; Bouchez, D.; Camilleri, C.; Costantino, P.; Vittorioso, P. Mutations in the Dof zinc finger genes DAG2 and DAG1 influence with opposite effects the germination of Arabidopsis seeds. Plant Cell 2002, 14, 1253-1263. [CrossRef]

65. Park, D.H.; Lim, P.O.; Kim, J.S.; Cho, D.S.; Hong, S.H.; Nam, H.G. The Arabidopsis COG1 gene encodes a Dof domain transcription factor and negatively regulates phytochrome signaling. Plant J. 2003, 34, 161-171. [CrossRef] [PubMed]

66. Papi, M.; Sabatini, S.; Bouchez, D.; Camilleri, C.; Costantino, P.; Vittorioso, P. Identification and disruption of an Arabidopsis zinc finger gene controlling seed germination. Genes Dev. 2000, 14, 28-33. [PubMed]

67. Ward, J.M.; Cufr, C.A.; Denzel, M.A.; Neff, M.M. The Dof transcription factor OBP3 modulates phytochrome and cryptochrome signaling in Arabidopsis. Plant Cell 2005, 17, 475-485. [CrossRef]

68. Wei, Z.; Yuan, T.; Tarkowská, D.; Kim, J.; Nam, H.G.; Novák, O.; He, K.; Gou, X.; Li, J. Brassinosteroid biosynthesis is modulated via a transcription factor cascade of COG1, PIF4, and PIF5. Plant Physiol. 2017, 174, 1260-1273. [CrossRef]

69. Renard, J.; Martínez-Almonacid, I.; Sonntag, A.; Molina, I.; Moya-Cuevas, J.; Bissoli, G.; Muñoz-Bertomeu, J.; Faus, I.; Niñoles, R.; Shigeto, J. PRX2 and PRX25, peroxidases regulated by COG1, are involved in seed longevity in Arabidopsis. Plant Cell Environ. 2020, 43, 315-326. [CrossRef] [PubMed]

70. Inoue, K.; Araki, T.; Endo, M. Circadian clock during plant development. J. Plant Res. 2018, 131, 59-66. [CrossRef] [PubMed]

71. Song, Y.H.; Ito, S.; Imaizumi, T. Flowering time regulation: Photoperiod-and temperature-sensing in leaves. Trends Plant Sci. 2013, 18, 575-583. [CrossRef]

72. Imaizumi, T.; Schultz, T.F.; Harmon, F.G.; Ho, L.A.; Kay, S.A. FKF1 F-box protein mediates cyclic degradation of a repressor of CONSTANS in Arabidopsis. Science 2005, 309, 293-297. [CrossRef] [PubMed]

73. Shim, J.S.; Kubota, A.; Imaizumi, T. Circadian clock and photoperiodic flowering in Arabidopsis: CONSTANS is a hub for signal integration. Plant Physiol. 2017, 173, 5-15. [CrossRef]

74. Xu, J.; Dai, H. Brassica napus Cycling Dof Factor1 (BnCDF1) is involved in flowering time and freezing tolerance. Plant Growth Regul. 2016, 80, 315-322. [CrossRef]

75. Rueda-Romero, P.; Barrero-Sicilia, C.; Gómez-Cadenas, A.; Carbonero, P.; Oñate-Sánchez, L. Arabidopsis thaliana DOF6 negatively affects germination in non-after-ripened seeds and interacts with TCP14. J. Exp. Bot. 2012, 63, 1937-1949. [CrossRef] [PubMed]

76. Yilmaz, A.; Mejia-Guerra, M.K.; Kurz, K.; Liang, X.; Welch, L.; Grotewold, E. AGRIS: The Arabidopsis gene regulatory information server, an update. Nucleic Acids Res. 2010, 39 (Suppl. 1), D1118-D1122. [CrossRef]

77. Yamamoto, M.P.; Onodera, Y.; Touno, S.M.; Takaiwa, F. Synergism between RPBF Dof and RISBZ1 bZIP activators in the regulation of rice seed expression genes. Plant Physiol. 2006, 141, 1694-1707. [CrossRef]

78. Mena, M.; Vicente-Carbajosa, J.; Schmidt, R.J.; Carbonero, P. An endosperm-specific DOF protein from barley, highly conserved in wheat, binds to and activates transcription from the prolamin-box of a native B-hordein promoter in barley endosperm. Plant $J$. 1998, 16, 53-62. [CrossRef]

79. Wu, J.; Chen, L.; Chen, M.; Zhou, W.; Dong, Q.; Jiang, H.; Cheng, B. The DOF-domain transcription factor ZmDOF36 positively regulates starch synthesis in transgenic maize. Front. Plant Sci. 2019, 10, 465. [CrossRef] [PubMed]

80. Fujimoto, S.; Matsunaga, S.; Yonemura, M.; Uchiyama, S.; Azuma, T.; Fukui, K. Identification of a novel plant MAR DNA binding protein localized on chromosomal surfaces. Plant Mol. Biol. 2004, 56, 225-239. [CrossRef]

81. Nan, H.; Ludlow, R.A.; Lu, M.; An, H. Genome-Wide Analysis of Dof Genes and Their Response to Abiotic Stress in Rose (Rosa chinensis). Front. Genet. 2021, 12, 168. [CrossRef]

82. Yang, G.; Gao, X.; Ma, K.; Li, D.; Jia, C.; Zhai, M.; Xu, Z. The walnut transcription factor JrGRAS2 contributes to high temperature stress tolerance involving in Dof transcriptional regulation and HSP protein expression. Bmc Plant Biol. 2018, 18, 1-14. [CrossRef] [PubMed]

83. Renau-Morata, B.; Molina, R.V.; Carrillo, L.; Cebolla-Cornejo, J.; Sánchez-Perales, M.; Pollmann, S.; Domínguez-Figueroa, J.; Corrales, A.R.; Flexas, J.; Vicente-Carbajosa, J. Ectopic expression of CDF3 genes in tomato enhances biomass production and yield under salinity stress conditions. Front. Plant Sci. 2017, 8, 660. [CrossRef] 
84. Lau, O.S.; Deng, X.W. Plant hormone signaling lightens up: Integrators of light and hormones. Curr. Opin. Plant Biol. 2010, 13, 571-577. [CrossRef] [PubMed]

85. Agarwal, P.; Jha, B. Transcription factors in plants and ABA dependent and independent abiotic stress signalling. Biol. Plant. 2010, 54, 201-212. [CrossRef]

86. Dar, N.A.; Amin, I.; Wani, W.; Wani, S.A.; Shikari, A.B.; Wani, S.H.; Masoodi, K.Z. Abscisic acid: A key regulator of abiotic stress tolerance in plants. Plant Gene 2017, 11, 106-111. [CrossRef]

87. Gasteiger, E.; Hoogland, C.; Gattiker, A.; Wilkins, M.R.; Appel, R.D.; Bairoch, A. Protein identification and analysis tools on the ExPASy server. In The Proteomics Protocols Handbook; Springer: Berlin/Heidelberg, Germany, 2005; pp. 571-607.

88. Nei, M.; Gojobori, T. Simple methods for estimating the numbers of synonymous and nonsynonymous nucleotide substitutions. Mol. Biol. Evol. 1986, 3, 418-426.

89. Hu, B.; Jin, J.; Guo, A.-Y.; Zhang, H.; Luo, J.; Gao, G. GSDS 2.0: An upgraded gene feature visualization server. Bioinformatics 2015, 31, 1296-1297. [CrossRef] [PubMed]

90. Törönen, P.; Medlar, A.; Holm, L. PANNZER2: A rapid functional annotation web server. Nucleic Acids Res. 2018, 46, W84-W88. [CrossRef] [PubMed]

91. Bolser, D.; Staines, D.M.; Pritchard, E.; Kersey, P. Ensembl plants: Integrating tools for visualizing, mining, and analyzing plant genomics data. In Plant Bioinformatics; Springer: Berlin/Heidelberg, Germany, 2016; pp. 115-140.

92. Krzywinski, M.; Schein, J.; Birol, I.; Connors, J.; Gascoyne, R.; Horsman, D.; Jones, S.J.; Marra, M.A. Circos: An information aesthetic for comparative genomics. Genome Res. 2009, 19, 1639-1645. [CrossRef] [PubMed]

93. Bray, N.L.; Pimentel, H.; Melsted, P.; Pachter, L. Near-optimal probabilistic RNA-seq quantification. Nat. Biotechnol. 2016, 34, 525-527. [CrossRef]

94. Gu, Z.; Eils, R.; Schlesner, M. Complex heatmaps reveal patterns and correlations in multidimensional genomic data. Bioinformatics 2016, 32, 2847-2849. [CrossRef] 